\title{
THE FAINT END OF THE LUMINOSITY FUNCTION AND LOW SURFACE BRIGHTNESS GALAXIES
}

\author{
Margaret J. Geller ${ }^{1}$, Antonaldo Diaferio ${ }^{2,3,4}$, Michael J. Kurtz ${ }^{1}$, Ian P. Dell'Antonio ${ }^{5}$, And Daniel G. Fabricant ${ }^{1}$ \\ ${ }^{1}$ Smithsonian Astrophysical Observatory, 60 Garden Street, Cambridge, MA 02138, USA; mgeller@ cfa.harvard.edu, \\ mkurtz@cfa.harvard.edu, dfabricant@cfa.harvard.edu \\ 2 Dipartimento di Fisica Generale 'Amedeo Avogadro', Università degli Studi di Torino, via P. Giuria 1, 10125 Torino, Italy \\ ${ }^{3}$ INFN, Sezione di Torino, via P. Giuria 1, 10125 Torino, Italy \\ ${ }^{4}$ Harvard-Smithsonian Center for Astrophysics, 60 Garden Street, Cambridge, MA 02138, USA; adiaferio@ @fa.harvard.edu \\ ${ }^{5}$ Department of Physics, Brown University, Box 1843, Providence, RI 02912, USA; ian@ het.brown.edu \\ Received 2011 July 12; accepted 2012 February 13; published 2012 March 16
}

\begin{abstract}
Smithsonian Hectospec Lensing Survey (SHELS) is a dense redshift survey covering a $4 \mathrm{deg}^{2}$ region to a limiting $R=20.6$. In the construction of the galaxy catalog and in the acquisition of spectroscopic targets, we paid careful attention to the survey completeness for lower surface brightness dwarf galaxies. Thus, although the survey covers a small area, it is a robust basis for computation of the slope of the faint end of the galaxy luminosity function to a limiting $M_{R}=-13.3+5 \log h$. We calculate the faint-end slope in the $R$ band for the subset of SHELS galaxies with redshifts in the range $0.02 \leqslant z<0.1$ SHELS $_{0.1}$. This sample contains 532 galaxies with $R<20.6$ and with a median surface brightness within the half-light radius of $\mathrm{SB}_{50, R}=21.82 \mathrm{mag} \operatorname{arcsec}^{-2}$. We used this sample to make one of the few direct measurements of the dependence of the faint end of the galaxy luminosity function on surface brightness. For the sample as a whole the faint-end slope, $\alpha=-1.31 \pm 0.04$, is consistent with both the Blanton et al. analysis of the Sloan Digital Sky Survey and the Liu et al. analysis of the COSMOS field. This consistency is impressive given the very different approaches of these three surveys. A magnitude-limited sample of 135 galaxies with optical spectroscopic redshifts with mean half-light surface brightness, $\mathrm{SB}_{50, R} \geqslant 22.5$ mag $\operatorname{arcsec}^{-2}$ is unique to SHELS $_{0.1}$. The faint-end slope is $\alpha_{22.5}=-1.52 \pm 0.16$. SHELS $S_{0.1}$ shows that lower surface brightness objects dominate the faint-end slope of the luminosity function in the field, underscoring the importance of surface brightness limits in evaluating measurements of the faint-end slope and its evolution.
\end{abstract}

Key words: galaxies: distances and redshifts - galaxies: dwarf - galaxies: fundamental parameters - galaxies: luminosity function, mass function

Online-only material: color figures, machine-readable and VO tables

\section{INTRODUCTION}

The faint end of the galaxy luminosity function is a fundamental constraint on theories of galaxy formation. All determinations of the low-luminosity slope are dramatically shallower than the predicted mass function of dark matter halos. Baryonic physics appears to be the key to resolving this discrepancy.

Physical processes possibly relevant to the faint-end slope include the gas cooling time (White \& Rees 1978), suppression by photoionization of low-mass galaxy formation (Benson et al. 2002), "feedback" mechanisms (Benson et al. 2003), merging, and tidal stripping. Benson et al. (2002, 2003) show that various combinations of these processes lead to very different faintend slopes. These differences can be a function of galaxy environment.

Measuring the slope of the faint end of the galaxy luminosity function, $\alpha$, remains an unresolved observational challenge. For the "field" luminosity function deeper redshift surveys covering increasing areas of the sky provide a route to better and better constraints. However, in any magnitude-limited redshift survey the least luminous galaxies occupy a small volume. Because luminosity and surface brightness are strongly correlated, neither the detection nor the spectroscopy of the lowest luminosity galaxies is trivial. Thus incompleteness at the faint end is a frustrating and serious issue.

Disney \& Phillipps (1983) and McGaugh (1996) emphasized the systematic biases resulting from failure to include low surface brightness (LSB) galaxies in the determination of the luminosity function. Sprayberry et al. (1997) made an early measurement of the impact of LSB galaxies on the determination of the field luminosity function. Their analysis, based on a catalog of LSB galaxies derived from Automatic Plate Measuring scans (Impey et al. 1996), demonstrates that the inclusion of LSB galaxies substantially steepens the field luminosity function. They obtained a faint-end slope $\alpha=-1.46$ in the $B$ band. For active star-forming galaxies in the TwoDegree Field redshift survey based on $b_{J}$ photometry, Madgwick et al. (2002) obtained a faint-end slope of $\alpha=-1.5$.

Also in the $B$ band, Driver et al. (2005) analyze the carefully constructed Millennium Galaxy Catalog and derive a faint-end slope for the global luminosity function of $\alpha=-1.13 \pm 0.02$. They examine the relationship between luminosity and surface brightness for their sample and conclude that the surface brightness distribution is broader for less luminous objects. Driver et al. (2005) show that the faint-end slope of the luminosity function is sensitive to the limiting surface brightness of the survey. Earlier $B$-band work by Cross \& Driver (2002) had yielded an even shallower faint-end slope and indicated robustness to surface brightness issues.

Blanton et al. (2005a) made a major step toward measuring and understanding the behavior of the field luminosity function at low luminosity across all of the Sloan Digital Sky Survey (SDSS) photometric bands. They construct and analyze a lowredshift sample of galaxies from the SDSS to place constraints on $\alpha$. Blanton et al. (2005a) caution that their sample is not necessarily complete at least in part because the SDSS was not optimized for this application and they carefully simulate their surface brightness completeness. They measure $\alpha \sim-1.3$ 
in the $r$ band. Interestingly, this result is similar to the faintend slope of spectroscopically determined cluster and group luminosity functions extending to comparably low luminosities (e.g., Mahdavi et al. 2005; Rines \& Geller 2008). They also argue that missing LSB galaxies could steepen the slope to $\alpha \sim-1.5$. Like Sprayberry et al. (1997), Blanton et al. (2005a) conclude that a majority of the faint galaxy population is blue, low concentration, and LSB.

Here we use a deep complete redshift survey with $R \leqslant 20.6$, the Smithsonian Hectospec Lensing Survey (SHELS hereafter; Geller et al. 2005, 2010) covering $4 \mathrm{deg}^{2}$ of the sky to constrain the value of $\alpha$. The galaxy catalog derives from the Deep Lens Survey (DLS hereafter; Wittman et al. 2006) which reaches a $1 \sigma$ surface brightness limit of $\mu_{R}=28.7 \mathrm{mag} \operatorname{arcsec}^{-2}$. We constrain $\alpha$ for redshifts $z \leqslant 0.1$ focusing on the contribution of blue, LSB galaxies.

We review the SHELS survey in Section 2. Section 2 contains a discussion of the survey completeness as a function of surface brightness. We discuss the magnitude-surface brightness relation for the survey. In Section 3, we display some of the lowest luminosity objects in our sample and we derive the luminosity function for the sample segregated by surface brightness. In Section 4 we compare the faint-end slope of our luminosity function with the COSMOS faint-end slope based on photometric redshifts (Liu et al. 2008). We conclude in Section 5.

\section{THE DATA}

We use two ambitious surveys to explore the faint end of the galaxy luminosity function. Generally low-luminosity galaxies have LSB. We take advantage of the properties of the two surveys that particularly enable access to these LSB objects.

The DLS (Wittman et al. 2006) is an NOAO key program covering $20 \mathrm{deg}^{2}$ in five separate fields; we use the $4 \mathrm{deg}^{2}$ F2 field at $\alpha=09^{\mathrm{h}} 19^{\mathrm{m}} 32^{\mathrm{s}} .4$ and $\delta=+30^{\circ} 00^{\prime} 00^{\prime \prime}$. The DLS photometric data were acquired in a $5 \mathrm{hr}$ integration on the Mayall $4 \mathrm{~m}$ in $<0$.'9 seeing and reaching a $1 \sigma$ limit for source detection in $R$ of 28.7 mag $\operatorname{arcsec}^{-2}$ is a good basis for identifying LSB galaxy candidates. We describe our approach to this issue in Section 2.1.

SHELS (Geller et al. 2005, 2010) is a redshift survey covering the F2 field to a limiting apparent magnitude $R=20.6$. SHELS is $98 \%$ complete to $R=20.3,96 \%$ complete to $R=20.6$. SHELS contains 541 galaxies with $R \leqslant 20.6$ at $z \leqslant 0.1$ where we can examine the behavior of the luminosity functions for the lowest luminosity galaxies. We made a concerted attempt to measure a redshift for each of the lowest surface brightness candidates in the photometric catalog. We describe the completeness of the entire SHELS redshift survey as a function of surface brightness in Section 2.2 .

SHELS covers a small field with deep photometry and spectroscopy. The SDSS covers a very wide field to a much brighter limiting apparent magnitude. We contrast the two surveys in Section 2.3.

\subsection{Photometry}

Photometric observations of F2 were made with the MOSAIC I imager (Muller et al. 1998) on the KPNO Mayall $4 \mathrm{~m}$ telescope between 1999 November and 2004 November. The DLS-selected all fields including F2 to exclude apparently bright nearby galaxies and to avoid known rich clusters with redshift $z \lesssim 0.1$. Even though rich clusters are rare, this selection biases the density at $z \lesssim 0.1$ toward values below the average for the local universe as a whole. We revisit this issue in Section 4.
The $R$-band exposures are the basis for the galaxy catalog in F2. The effective exposure is $14,500 \mathrm{~s}$. Wittman et al. (2006) describe the imaging reduction pipeline. The $1 \sigma$ limit for source detection in $R$ is $28.7 \mathrm{mag} \operatorname{arcsec}^{-2}$. To this limit there are 45 sources $\operatorname{arcmin}^{-2}$. The automatic object identification algorithm produces a complete catalog of objects with surface brightness $\mu_{50, R} \leqslant 27.0$ within the half-light radius.

We constructed a galaxy catalog from the $R$-band source list; we base our luminosity function computation on this $R$-band catalog. We selected galaxy candidates with Kron-Cousins $R \leqslant$ 20.6 for spectroscopic observation. The magnitudes are extrapolated total magnitudes; they are extrapolated from isophotal magnitudes within the limiting $28.7 \mathrm{mag} \operatorname{arcsec}^{-2}$ isophote.

Nearly, all of the galaxy candidates with $R \leqslant 20.6$ from the DLS also have SDSS photometry; there are only 104 DLS galaxy candidates without SDSS photometry. Many of these objects are not resolved by the SDSS and thus they have a broad range in surface brightness. We do spectroscopy for these objects as for all other galaxy candidates. When we compare the DLS with the SDSS, we estimate $r$-band magnitudes from the $R$-band DLS photometry and from our spectroscopy.

Figure 1 shows the classification diagram we use to select galaxies from the DLS object list. The upper left-hand panel shows all of the 302,574 objects in F2 with $R<22.5$. We use the difference between the magnitude within our 1".5 fiber, $R_{1^{\prime \prime} .5}$, and the total magnitude $R$ as a discriminant. The upper right-panel shows (dark dots) all of the SHELS galaxies with redshifts. There are 15,652 redshifts for galaxies with $R<21$ and 12,783 for galaxies with $R<20.6$ (vertical green line).

Objects with the smallest $R_{1^{\prime \prime} .5}-R$ are mostly stars; objects with larger $R_{1 \prime}{ }_{1.5}-R$ that lie above the dense galaxy locus generally result from background fluctuations and various artifacts around bright stars and/or diffraction spikes.

To construct the catalog for SHELS spectroscopy, we examined all of the 33,038 objects with $R \leqslant 20.6$ visually to remove the obvious artifacts. The initial catalog of 33,038 objects is complete to $\mu_{50, R}=27.0$ at the expense of including artifacts. We conservatively included some apparently stellar objects in the observing list, including any of these objects classified as a galaxy by the SDSS; our spectroscopy then showed that $\sim 5 \%$ of the spectroscopically observed objects with $R \gtrsim 19.5$ are, in fact, stars.

Very few of the objects that lie above the dense locus of SHELS points are candidate galaxies; we included all objects that were not obviously noise or other artifacts in the spectroscopic observing list. The galaxy candidates are all small compared to the largest angular size objects contained in the catalog. The galaxy candidates are well away from the photometric thresholds in Figure 1, implying little or no bias against detection of large LSB objects in the photometric catalog.

\subsection{Spectroscopy}

We acquired spectra for the objects with the Hectospec (Fabricant et al. 1998, 2005) on the MMT from 2004 April 13 to 2007 April 20. The Hectospec observation planning software (Roll et al. 1998) enables efficient acquisition of a magnitudelimited sample. We made a concerted effort to acquire spectra for the lowest surface brightness objects.

The SHELS spectra cover the wavelength range 3700-9100 with a resolution of $\sim 6 \AA$. Exposure times ranged from 0.75 to $2 \mathrm{hr}$. The $2 \mathrm{hr}$ exposures are adequate to yield a redshift even for the lowest surface brightness objects. The lowest surface 

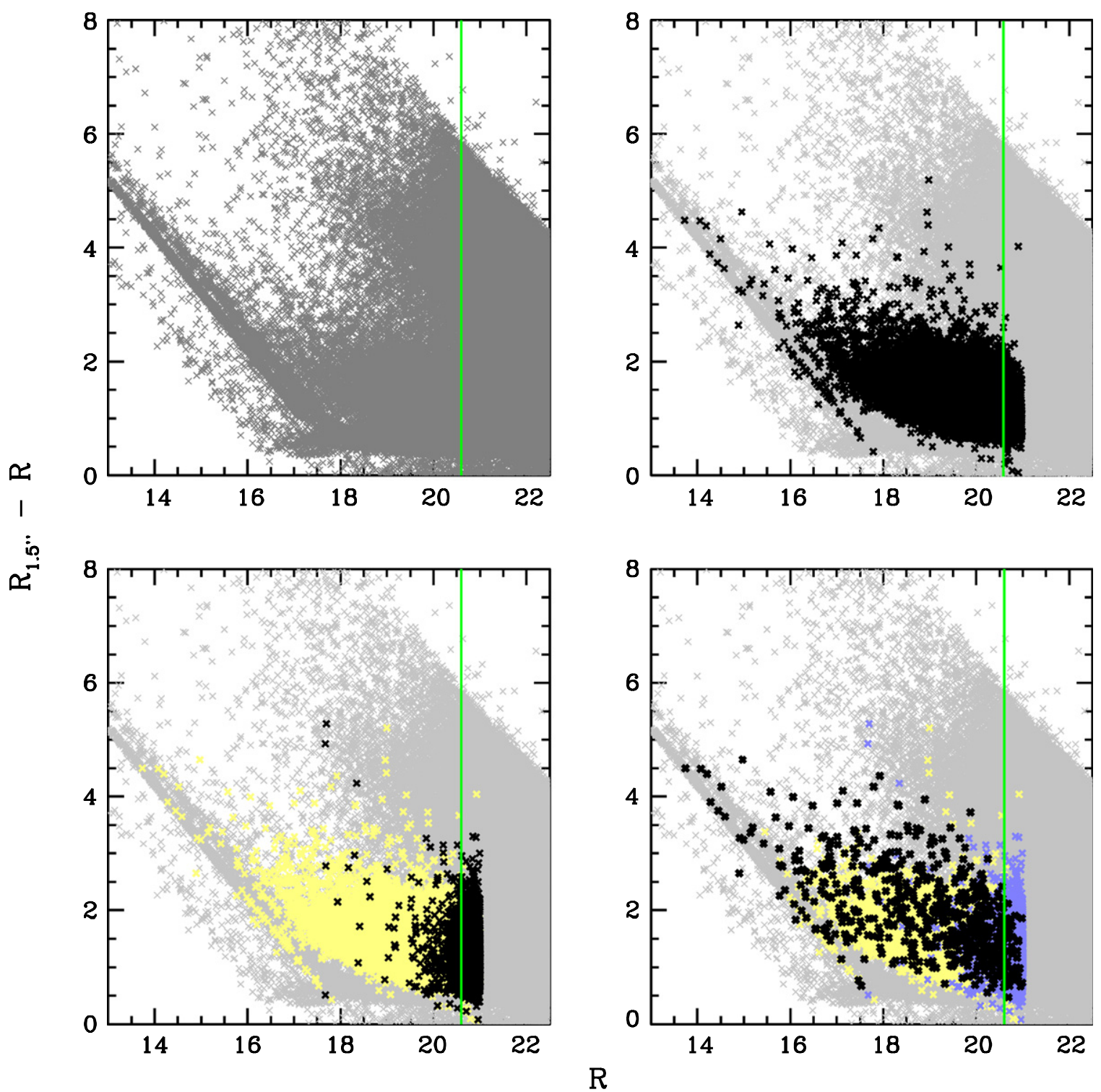

Figure 1. SHELS galaxy selection from DLS photometry. $R_{1^{\prime \prime}}$ is the DLS magnitude within the $1^{\prime \prime} .5$ Hectospec fiber aperture. $R$ is the total DLS apparent magnitude. The vertical green line shows the SHELS survey limit $R=20.6$. Gray points show all of the objects detected in the DLS survey; the black points (upper right panel) show all of the galaxies with SHELS spectra and $R \leqslant 21$. Most of the "objects" at large $R_{1^{\prime \prime} 5}-R$ (above the main locus of objects with SHELS spectra) and with $R<20.6$ are artifacts. In the lower two panels, yellow points represent all galaxies with measured redshifts. On the lower left, black points represent all galaxy candidates with $R \leqslant 21$ and without a measured redshift. In the lower right, black points represent SHELS $\mathrm{S}_{0.1}$ galaxies with redshift $z<0.1$, blue points are galaxy candidates without a redshift.

(A color version of this figure is available in the online journal.)

brightness objects in the survey required the longer integrations. We reduced the data with the standard Hectospec pipeline (Mink et al. 2007) and derived redshifts with RVSAO (Kurtz \& Mink 1998) with templates constructed for this purpose (Fabricant et al. 2005). Our 1468 unique pairs of repeat observations imply a mean internal error of $56 \mathrm{~km} \mathrm{~s}^{-1}$ for absorption-line objects and $21 \mathrm{~km} \mathrm{~s}^{-1}$ for emission-line objects (see also Fabricant et al. 2005).

For each spectrum we compute the stellar population age indicator, $D_{n} 4000$. This indicator is the ratio of flux in the 4000-4100 $\AA$ band to flux in the 3850-3950 $\AA$ band (Balogh et al. 1999); it is a measure of the strength of the $4000 \AA$ break. The rms scatter in our measurement of $D_{n} 4000$ is 0.086 . The internal error in $D_{n} 4000$ is only $4.5 \%$ based on our 1468 repeat measurements. A comparison of overlapping spectra with the SDSS yields a median ratio of 1.00 (Fabricant et al. 2008).
Following Woods et al. (2010) and Kauffmann et al. (2003), we use this indicator to segregate galaxies dominated by old and young stellar populations.

SHELS includes 13,362 galaxies to the limiting apparent magnitude, $R=20.6$. The integral completeness of the redshift survey to this limit is $96 \%$. Geometric constraints are responsible for the 579 objects without redshifts; they are mostly near the survey corners and edges. On average, Hectospec positionings revisit every region within the DLS field (except for the corners and edges) more than a dozen times. Thus we are minimally biased against close pairs and satellite galaxies.

The upper right-hand panel of Figure 1 shows $R_{1^{\prime \prime} .5}-R$ as a function of total magnitude $R$ for all objects with redshifts and $R<21$ (black points). The lower left-hand panel shows the objects with redshifts as yellow points and galaxy candidates without redshifts as black points. The concentration of objects 
Table 1

SHELS $_{0.1}$ Redshifts

\begin{tabular}{|c|c|c|c|c|c|c|c|c|c|}
\hline SHELS ID & SDSS ObjID & R.A.2000 & $\operatorname{Decl}_{.2000}$ & $R$ & $R$ Source $^{\mathrm{a}}$ & $z$ & $z$ Source $^{b}$ & $\begin{array}{c}\delta z \\
\left(\mathrm{~km} \mathrm{~s}^{-1}\right)\end{array}$ & $\begin{array}{c}\mu_{50, R} \\
\left(\mathrm{mag} \mathrm{arcsec}^{-2}\right)\end{array}$ \\
\hline $138.7082030+30.4863253$ & 587738947740697041 & $9: 14: 49.969$ & $30: 29: 10.771$ & 19.5084 & DLS & 0.0218412 & MMT & 32.3 & 22.5807 \\
\hline $138.7106146+30.1474856$ & 588017978876101272 & $9: 14: 50.547$ & 30:08:50.948 & 19.6469 & DLS & 0.0373713 & MMT & 47.1 & 24.1041 \\
\hline $138.7245438+30.2541523$ & 588017978876166576 & $9: 14: 53.891$ & $30: 15: 14.948$ & 18.8388 & DLS & 0.0236177 & MMT & 32.1 & 22.1238 \\
\hline $138.7254512+30.6434571$ & 588017979413103093 & $9: 14: 54.108$ & $30: 38: 36.446$ & 18.7908 & DLSm & 0.0238024 & MMT & 81.9 & 23.4231 \\
\hline $138.7268120+30.4084400$ & 587738947740696966 & $9: 14: 54.435$ & $30: 24: 30.384$ & 20.9377 & DLS & 0.0628633 & MMT & 40.9 & 22.8286 \\
\hline $138.7271334+29.2781541$ & 588017977802228132 & $9: 14: 54.512$ & $29: 16: 41.355$ & 19.4561 & DLS & 0.0206206 & MMT & 50.6 & 22.7012 \\
\hline $138.7289058+30.2024933$ & 588017978876166301 & $9: 14: 54.937$ & $30: 12: 08.976$ & 15.7759 & DLS & 0.0232745 & MMT & 18.4 & 20.6754 \\
\hline $138.7325010+30.0934152$ & 587738947203760418 & $9: 14: 55.800$ & $30: 05: 36.295$ & 18.3344 & DLS & 0.0238612 & MMT & 43.6 & 23.1907 \\
\hline $138.7353477+30.4341614$ & 587738947740696611 & $9: 14: 56.483$ & $30: 26: 02.981$ & 17.0162 & DLS & 0.0222926 & MMT & 34.3 & 21.69 \\
\hline $138.7358290+30.2827235$ & 588017978876166629 & $9: 14: 56.599$ & $30: 16: 57.805$ & 20.6605 & DLS & 0.0222285 & MMT & 47.6 & 23.1852 \\
\hline
\end{tabular}

Notes.

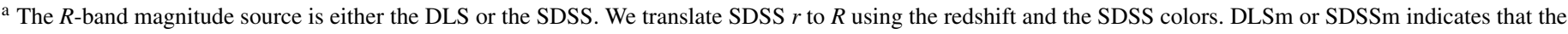
magnitudes required a detailed calculation outside the pipeline for the survey generally as a result of a nearby star and/or an artifact in the imaging data.

$\mathrm{b}$ The redshift $z$ is from the Hectospec on the MMT (MMT) or from the SDSS. The error $\delta z$ is also from the redshift source.

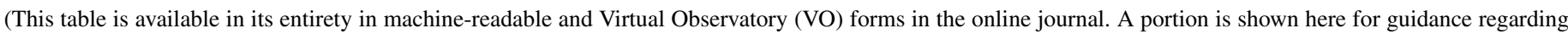
its form and content.)

without spectra toward faint magnitudes is obvious: there are 4238 objects with $R<21$ but only 579 with $R<20.6$.

The lower right-hand panel of Figure 1 shows the sample we use to study the faint end of the galaxy luminosity function, SHELS $_{0.1}$. SHELS $S_{0.1}$ includes all SHELS galaxies with $z<0.1$. There are 541 objects with $R<20.6$ and 482 with $R<20.3$. These galaxies tend to have larger $R_{1^{\prime \prime} 5}-R$ because they are nearby and hence larger on the sky. Most of the objects in the sample with large $R_{1^{\prime \prime} .5}-R$ are in this low-redshift subsample.

Table 1 contains the redshifts and $R$-band magnitudes for the SHELS $_{0.1}$ sample. The table includes redshifts we obtained for some galaxies fainter than the survey limit $R=20.6$ but with redshift $z \leqslant 0.1$. The table lists the SHELS identification (Column 1), the SDSS ObjID (Column 2), the right ascension (Column 3), the declination (Column 4), the $R$-band total magnitude (Column 5), the source for the magnitude (Column 6), the redshift (Column 7), the error in the redshift in $\mathrm{km} \mathrm{s}^{-1}$ (Column 8), and the observed mean $R$-band surface brightness within the half-light radius, $\mu_{50, R}$ (Column 9). Among these redshifts, 18 are from the SDSS; the rest are new Hectospec measurements.

Figure 2 shows the observed mean surface brightness within the half-light radius, $\mu_{50, R}$ as a function of the discriminant we use to construct the galaxy catalog, $R_{1}^{\prime \prime} 5-R$ (Figure 1 ). Gray points indicate all of the objects in the catalog. We also show (black points) the galaxies in the SHELS redshift survey (including galaxy candidates with or without a redshift and with $19<R<20.6$.). We can readily detect objects with $\mu_{50, R}>24$ mag $\operatorname{arcsec}^{-2}$ and we are complete to $\mu_{50, R}=27 \mathrm{mag} \mathrm{arcsec}^{-2}$, but galaxy candidates among these objects are rare in this local region of the universe. The lowest surface brightness galaxies we find have $\mu_{50, R} \sim 26 \mathrm{mag} \operatorname{arcsec}^{-2}$. It is interesting to note that all of the known dwarfs in the Local Group with $M_{R} \leqslant-13$ (the low-luminosity limit of our luminosity function; Section 3) have mean surface brightnesses within the range we can sample (e.g., Mateo 1998; Grebel et al. 2003).

Figure 3 shows the completeness of the entire SHELS redshift survey as a function of observed mean surface brightness within the half-light radius $\mu_{50, R}$. The completeness is simply the fraction of galaxies in the photometric catalog with measured redshifts as a function of observed $\mu_{50, R}$. The dashed histogram shows the fractional completeness for the sample of galaxies with $R<20.3$; the solid histogram refers to the sample with $R<20.6$. We took substantial care in repeat Hectospec fields to obtain the high level of completeness for $R<20.3$. Most of the incompleteness results from geometric constraints; we fail to sample the corners and edges of the field as well as we sample the central portion.

\subsection{Comparison of SHELS $S_{0.1}$ with the SDSS}

Blanton et al. (2005a) provide a benchmark for measurement of the faint end of the galaxy luminosity function in the local universe in the $r$ band. They carefully analyze the extensive NYU-VAGC catalog (Blanton et al. 2005b) of SDSS galaxies with $r<17.77$. Here we compare the central average surface brightness distributions and the surface brightness-luminosity relations for the NYU-VAGC and SHELS S.1 $_{0.1}$. Because Blanton et al. (2005a) study the $r$-band luminosity function, we focus on this comparison.

We first compare the surface brightness distribution of the SHELS $_{0.1}$ sample with the SDSS sample. Figure 4 shows the distribution of observed mean SDSS $r$-band surface brightness, $\mu_{50, r}$, for the galaxies in the SDSS sample with $z<0.05$ (solid gray histogram). The heavy solid line shows the observed $r$-band surface brightness distribution for the $\mathrm{SHELS}_{0.1}$ galaxies with $0.02<z<0.05$. We scale the SHELS $_{0.1}$ histogram by the relative area of the two surveys.

Figure 4 demonstrates two important aspects of SHELS S.1 $_{1}$ For observed $\mu_{50, r}$ around the peak SDSS sensitivity, SHELS $S_{0.1}$ has systematically fewer objects than the SDSS sample. This deficit in the SHELS $S_{0.1}$ survey reflects the a priori selection against nearby galaxies and clusters; the SHELS $_{0.1}$ region is underdense by selection. For observed $\mu_{50, r} \gtrsim 22$, the SHELS $S_{0.1}$ survey contains a relatively much larger number of objects than the SDSS. This difference results from the SHELS 0.1 redshift survey fainter magnitude limit and completeness for mostly blue LSB objects. The surface brightness distribution for the NYU-VAGC declines steeply over the surface brightness range 23-24 mag arcsec ${ }^{-2}$; the SHELS $_{0.1}$ distribution over this range is essentially flat.

Figure 5 shows the fraction of blue objects with $g-r<0.4$ and with $z<0.05$ as a function of $\mu_{50, r}$ for the SDSS (gray 


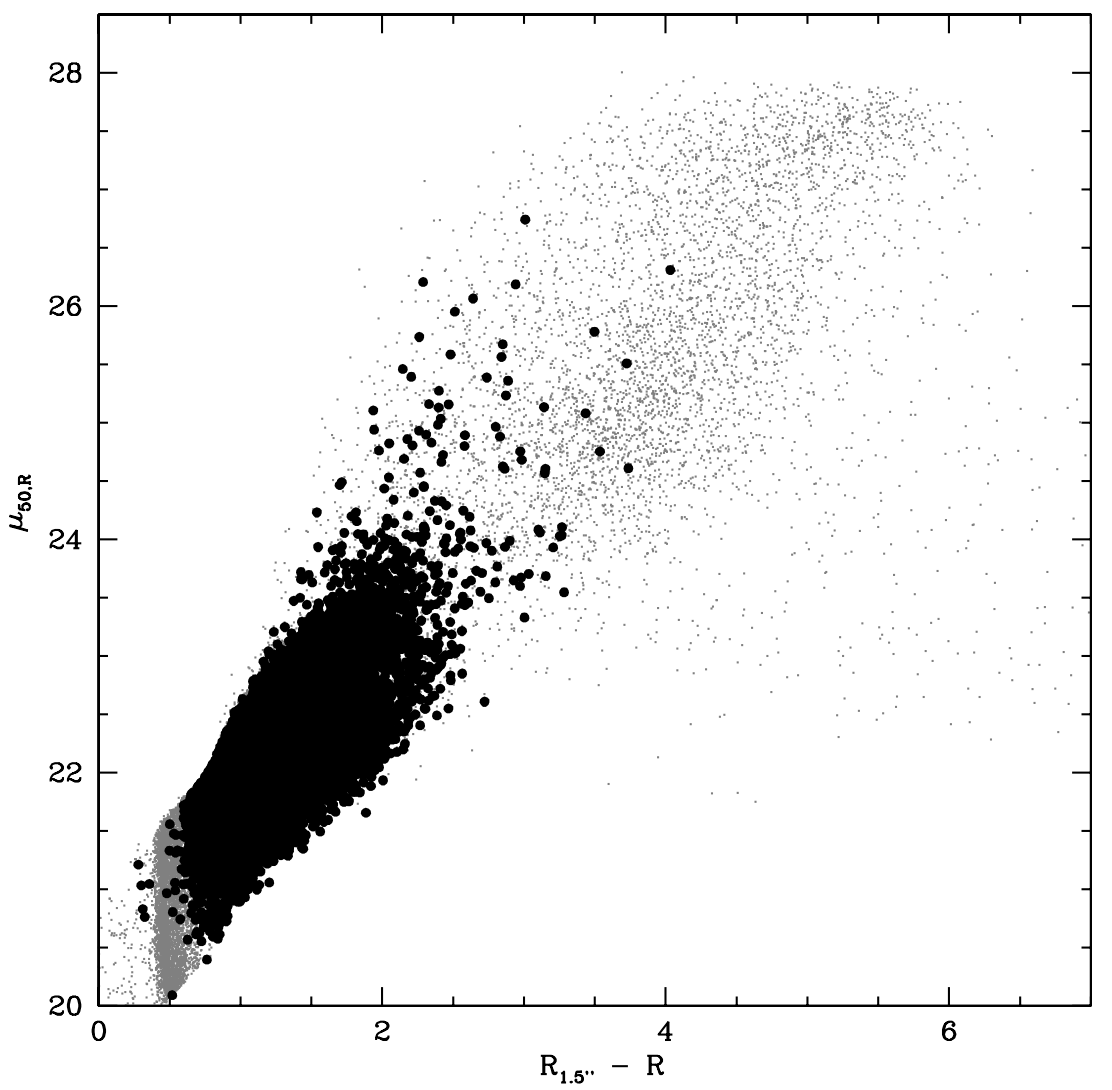

Figure 2. DLS observed mean surface brightness within the half-light radius, $\mu_{50, R}$ as a function of the magnitude difference $R_{1^{\prime \prime} 5}-R$ in the classification diagram of Figure 1. Gray points denote all of the DLS objects in the apparent magnitude range $19<R<20.6$. Black points denote all of the galaxy candidates (with or without a redshift). Note that the lowest surface brightness galaxies are all above the survey limit and that most of the low surface brightness objects are artifacts.

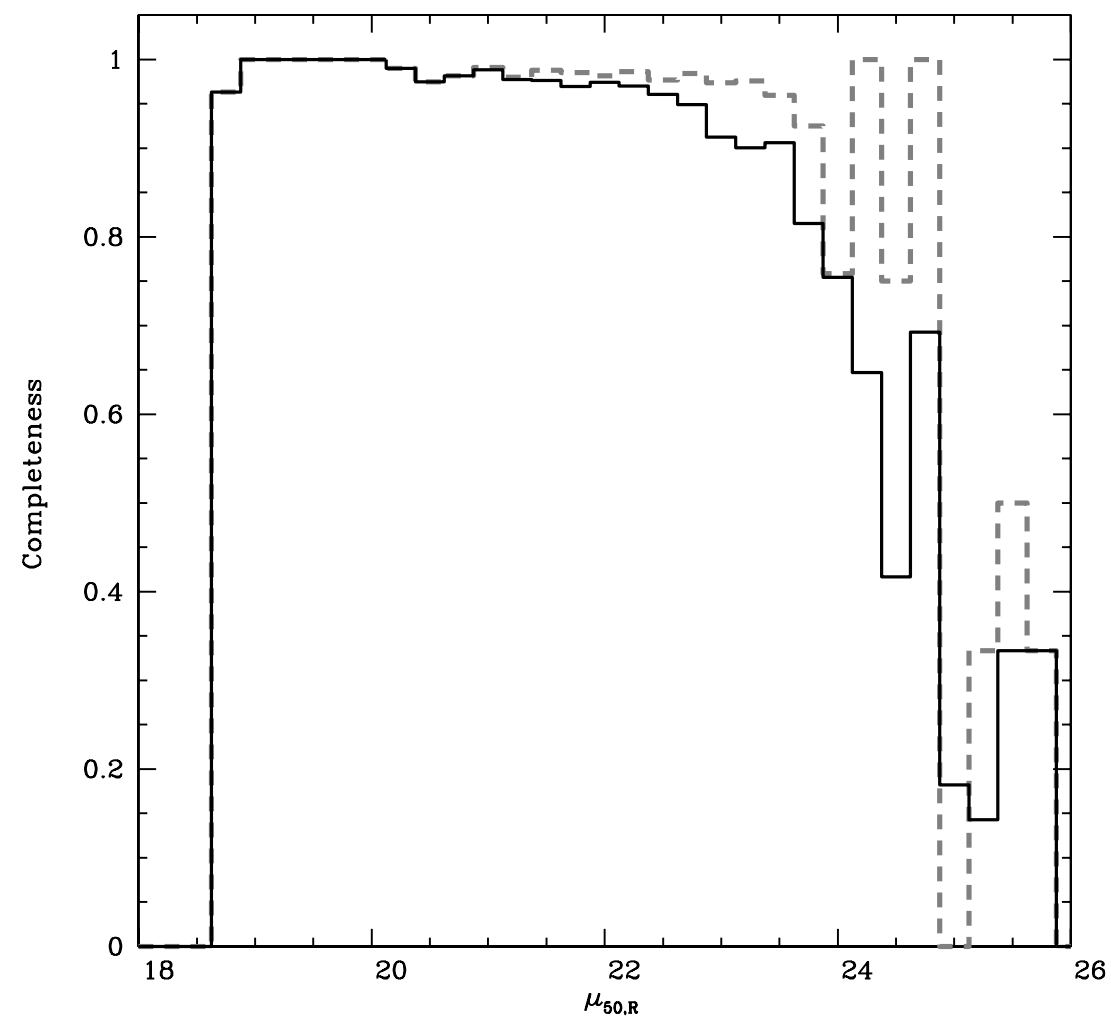

Figure 3. SHELS redshift survey completeness as a function of observed surface brightness, $\mu_{50, R}$ for galaxies with $R<20.3$ (dashed histogram) and for galaxies with $R<20.6$ (solid histogram) 


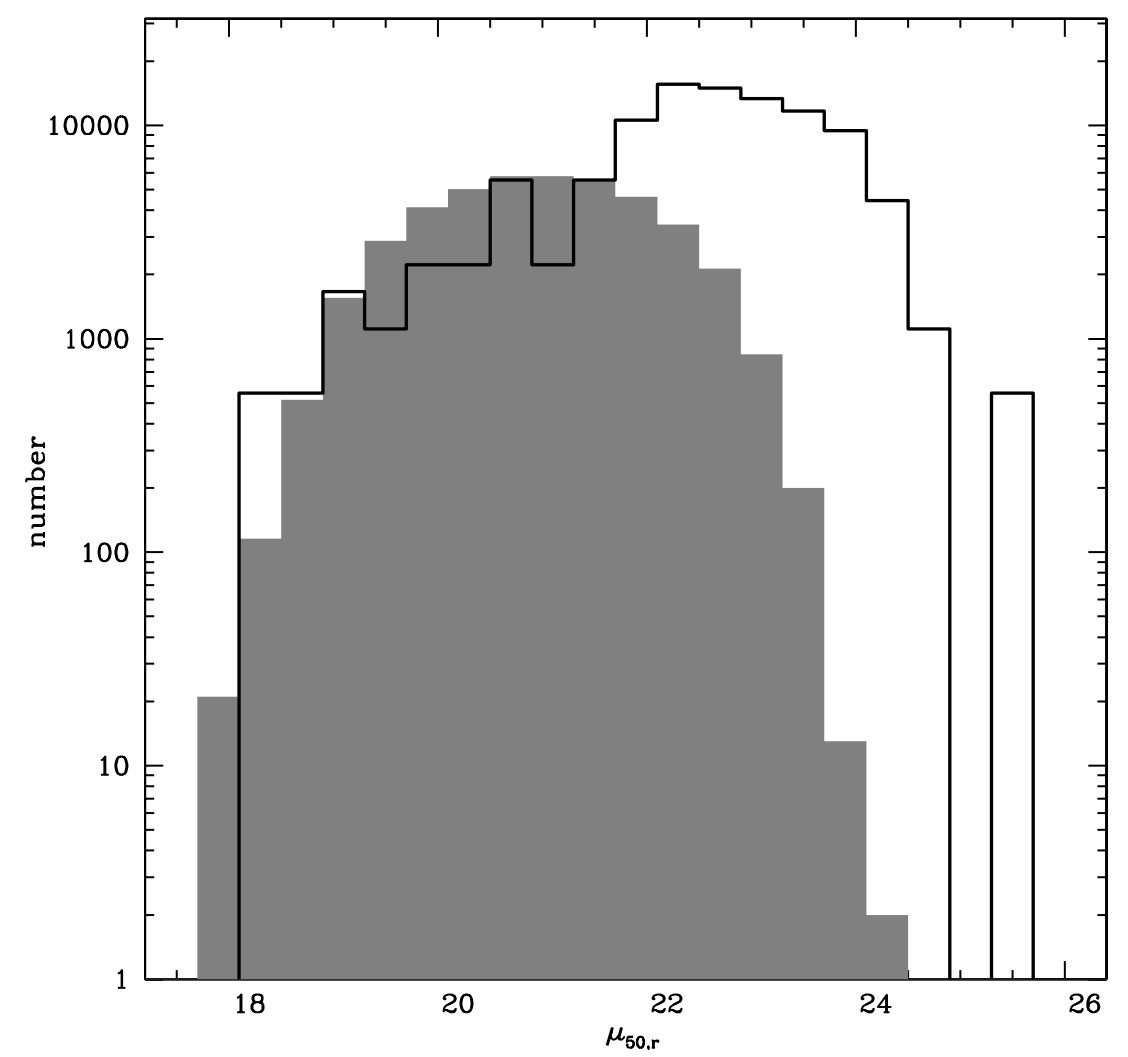

Figure 4. Comparison of the observed surface brightness distribution for the SDSS sample (solid gray histogram) of Blanton et al. (2005a) with galaxies in SHELS . $_{0.1}$ that have $z<0.05$ (heavy black line). $\mu_{50, r}$ is the SDSS observed mean $r$-band surface brightness within the half-light radius. The vertical axis shows the actual number of SDSS galaxies; we scale the SHELS $0.1 z<0.05$ sample by the relative areal coverage of the two surveys. Note the relatively larger representation of low surface brightness galaxies in the SHELS $0.1 z<0.05$ sample.

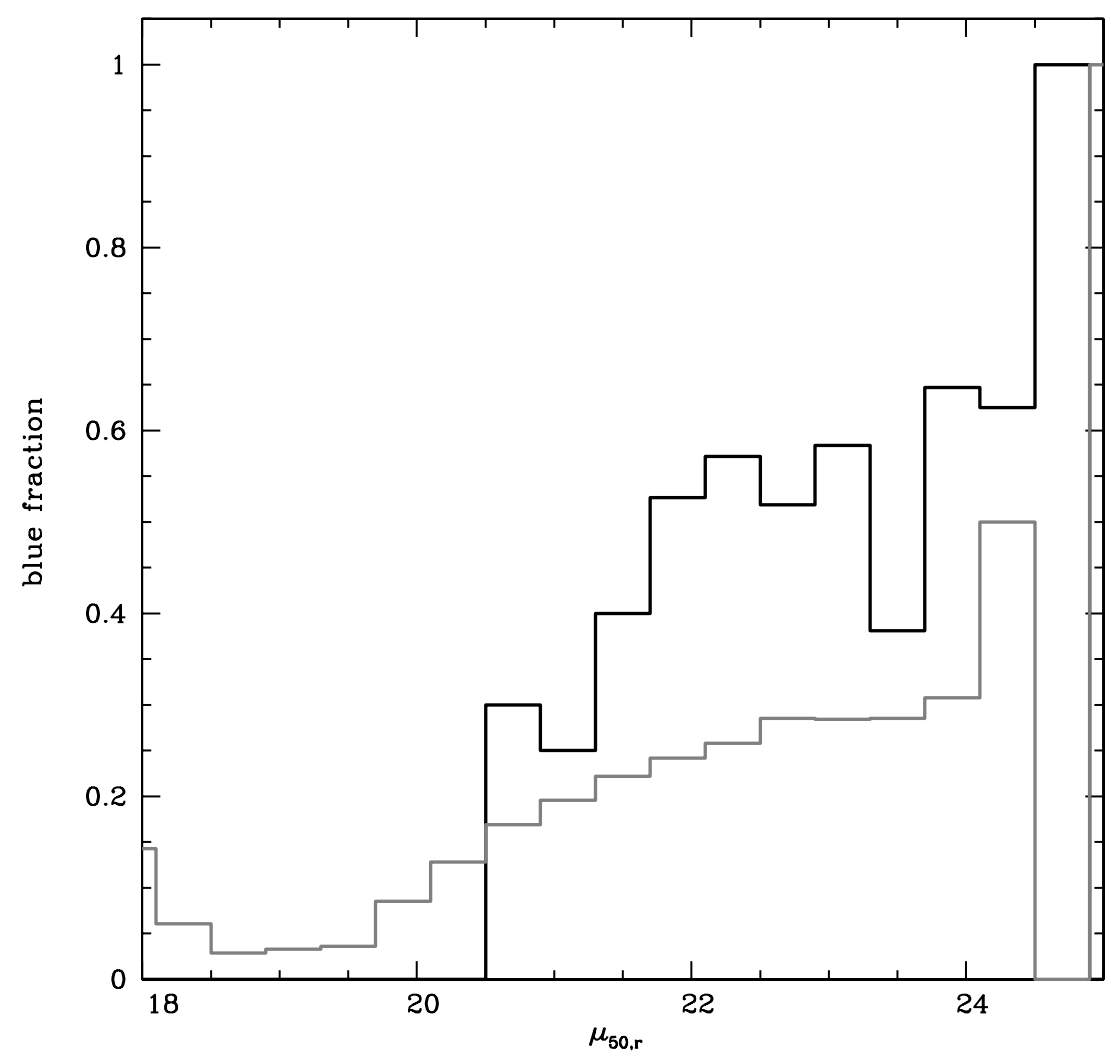

Figure 5. Fractions of galaxies with $g-r<0.4$ in Figure 4. The gray histogram shows the SDSS blue fraction; the black histogram shows the fraction for SHELS 0.1 galaxies with $0.02<z<0.05$. Note the greater blue fraction in the SHELS $0.1 z<0.05$ sample. 


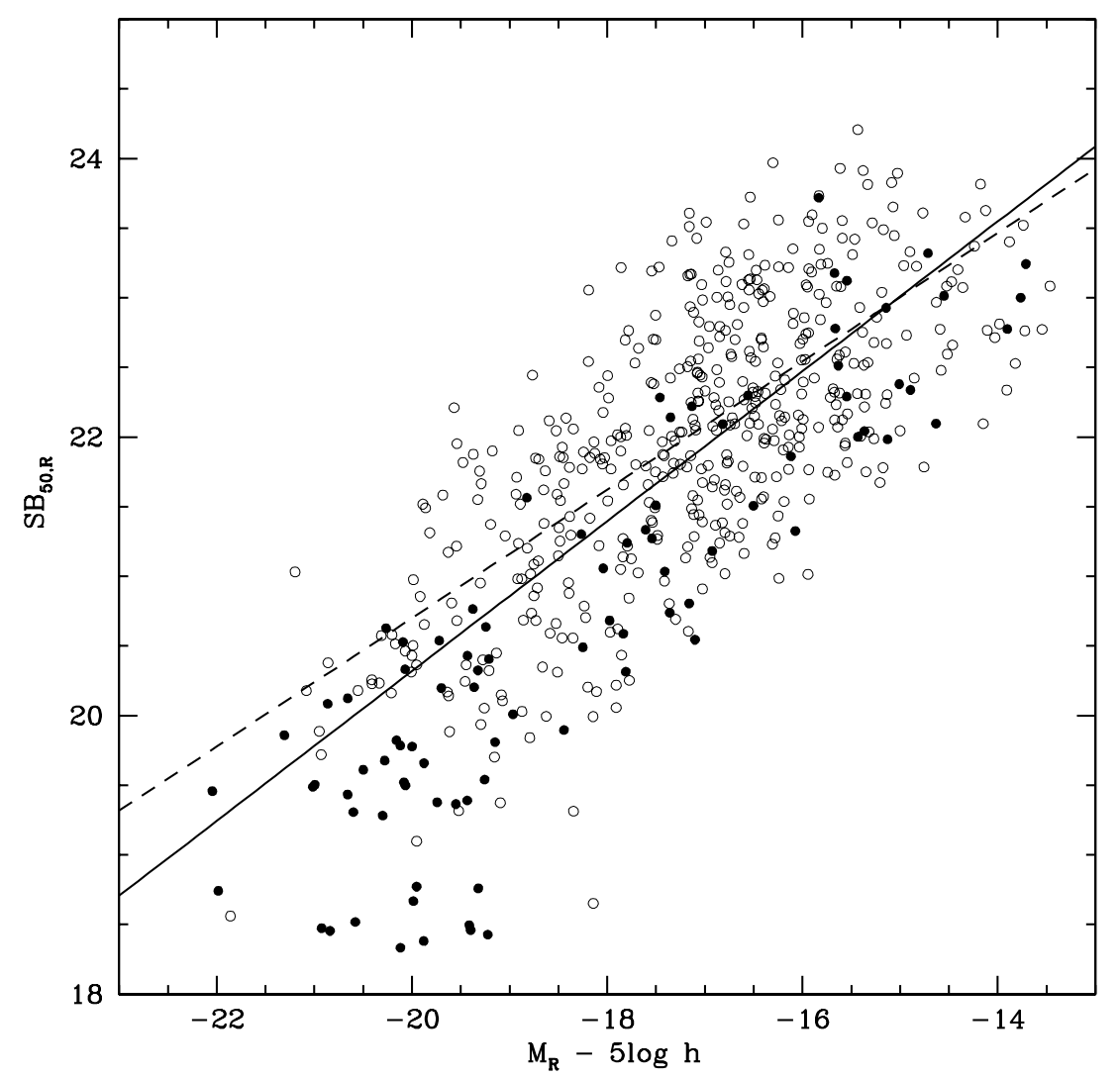

Figure 6. Mean rest-frame surface brightness, $\mathrm{SB}_{50, R}$ as a function of absolute magnitude, $M_{R}$ for $\mathrm{SHELS}_{0.1}$. The solid line shows the relation between the two quantities derived from a Bayesian analysis for the full SHELS $\mathrm{S}_{0.1}$ sample; the dashed line shows the relation for the galaxies with $D_{n} 4000<1.44$. Solid dots denote galaxies with $D_{n} 4000 \geqslant 1.44$; open circles denote objects with $D_{n} 4000<1.44$.

histogram) and for SHELS $_{0.1}$; the blue fraction is substantially larger in SHELS $_{0.1}$.

Because the SDSS has a substantial (known) incompleteness for $\mu_{50, r} \gtrsim 23$, Blanton et al. (2005a) model the incompleteness based on an extrapolation of the relationship between surface brightness and absolute magnitude for galaxies with $M_{r}<$ -18.0 . We can examine the relationship between the restframe surface brightness $\mathrm{SB}_{50, R}$ within the half-light radius and the absolute magnitude $M_{R}$ in SHELS $_{0.1}$ and thus test the Blanton et al. (2005a) relationship over a larger range in absolute magnitude. For comparison with Blanton et al. (2005a), the median $R-r=0.279$ for SHELS $_{0.1}$ galaxies.

The absolute $R$-band magnitude is

$$
M_{R}=m_{R}-5 \log d_{L}-25.0-k_{R}(z),
$$

where $d_{L}$ is the luminosity distance in Mpc for a Hubble constant $H=100 h \mathrm{~km} \mathrm{~s}^{-1} \mathrm{Mpc}^{-1}$ and $k_{R}(z)$ is the $R$-band $k$-correction derived according to the procedure in Westra et al. (2010). We use the Hubble constant normalized to 100 for easy comparison with Blanton et al. (2005a) and others.

The rest-frame surface brightness is

$$
\mathrm{SB}_{50, R}=\mu_{50, R}-10 \log _{10}(1+z)-k_{R}(z) .
$$

This equation implies that, for example, a typical survey galaxy with $\mu_{50, r}=25.0$ (at the LSB limit of Figure 5) has $\mathrm{SB}_{50, R} \simeq 24.3$ at the $z=0.1$ survey limit.

Figure 6 shows the relation between $\mathrm{SB}_{50, R}$ and absolute magnitude $M_{R}$. There is only one galaxy in the survey that lands outside the plot limits. It has $\mathrm{SB}_{50, R}=25.1$ and is obviously contaminated by a nearby bright star; we thus omit it.

We use a Bayesian approach to quantify the correlation between $\mathrm{SB}_{50, R}$ and absolute magnitude $M_{R}$. This approach is similar to the one used by Blanton et al. (2005a) but not identical. $\mathrm{SB}_{50, R}$ and $M_{R}$ clearly do not have a one-to-one relation, but, at fixed $M_{R}, \mathrm{SB}_{50, R}$ is distributed according to some probability density distribution (PDF), $p\left(\mathrm{SB}_{50, R} \mid M_{R}\right)$. In the Bayesian approach, we can infer the parameters of this PDF. We do not need to assume that the spread originates from random measurement errors around the ideal relation $\mathrm{SB}_{50, R}=a+b M_{R}$, as in a standard linear regression. In addition, unlike the usual fitting technique, we can model the uncertainties in the individual measures as random variates.

We assume that $\mathrm{SB}_{50, R}$ is normally distributed around the mean $\left\langle\mathrm{SB}_{50, R}\right\rangle=a+b M_{R}$ with variance $\sigma_{\text {int }}^{2}$. We then need to determine the three parameters $a, b$, and $\sigma_{\text {int }}$ and their PDFs. We assume flat priors for both $a$ and $b$. For the inverse of the variances of the individual measures, $1 / \sigma_{\mathrm{SB}_{50 . R}}{ }^{2}$ and $1 /{\sigma_{M_{R}}}^{2}$, we adopt the usual assumption that they are random variates drawn from a gamma distribution with large variance (e.g., Andreon \& Hurn 2010). This choice is appropriate for quantities that are positively defined and provides basically flat priors for the uncertainties.

We use the free software JAGS developed by Martyn Plummer $^{6}$ to run Markov chain Monte Carlo simulations. We estimate the PDFs of our three parameters by running $3 \times 10^{5}$ iterations. For the full sample SHELS $S_{0.1}$ we find $a=31.08_{-0.37}^{+0.36}$,

\footnotetext{
6 www-fis.iarc.fr/ martyn/software/jags/
} 

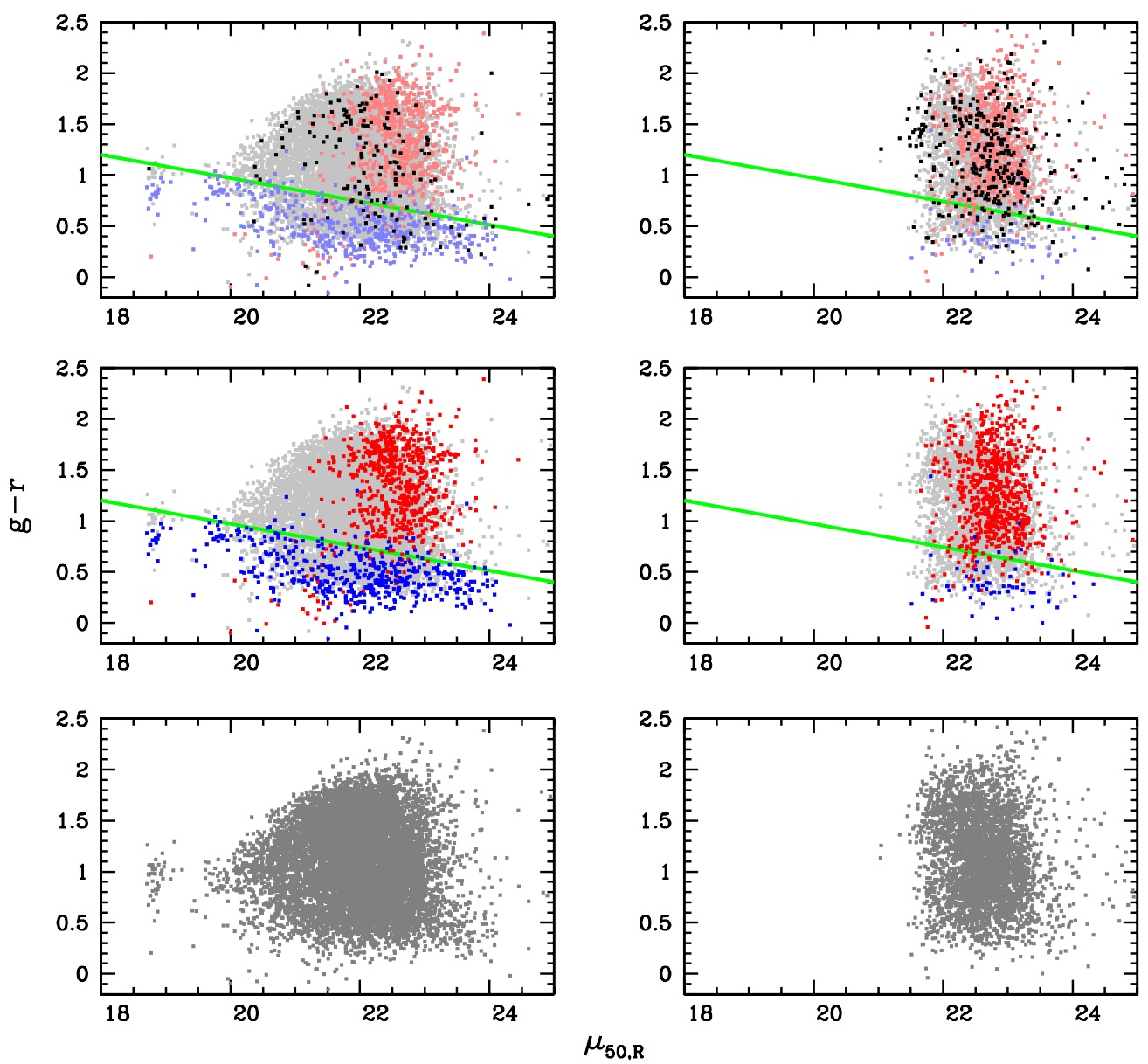

Figure 7. SDSS $g-r$ color as a function of DLS observed surface brightness, $\mu_{50, R}$ for SHELS. This plot is the basis for an estimate of the completeness of SHELS ${ }_{0.1}$. The left column applies to galaxies with $R<20.3$.; the right-hand column applies to galaxies with $20.3 \leqslant R<20.6$. The bottom panels show all galaxies in the magnitude range (gray points). The central panels show the low- and high-redshift portions of the sample: blue points represent galaxies with $z<0.1$ and red points represent galaxies with $z>0.5$. Not surprisingly the galaxies in the $20.3 \leqslant R<20.6$ interval are mostly at $z>0.1$ (gray and red points in the upper panel). Most of the low-redshift sample lies below the green line. Black points in the upper panels show all of the objects in the photometric survey without a redshift; only a few points overlap the color-surface brightness range spanned by the low-redshift sample indicated in blue.

(A color version of this figure is available in the online journal.)

$b=0.538_{-0.021}^{+0.020}, \sigma_{\text {int }}=0.791_{-0.025}^{+0.025}$. Figure 6 shows the sample and the result of the Bayesian analysis of the entire sample (solid line).

To compare more closely with the analysis of Blanton et al. (2005a), we examine the $\mathrm{SB}_{50, R}-M_{R}$ relation omitting the redder objects. Rather than explicitly using color to segregate the galaxy populations, we use the redshift-independent spectroscopic indicator $D_{n} 4000$. Woods et al. (2010) and Kauffmann et al. (2003) show that the distribution of this indicator is bimodal and that it can be used to segregate galaxies with younger stellar populations (generally blue objects) from those dominated by an old stellar population (generally red objects). At low-redshift segregation by $D_{n} 4000$ is essentially equivalent to segregation by $g-r$. Woods et al. (2010) divide their sample at the local minimum between the two peaks, $D_{n} 4000=1.44$.

For the 431 blue objects with $D_{n} 4000<1.44$, we find $a=29.92_{-0.39}^{+0.40}, b=0.461_{-0.023}^{+0.023}, \sigma_{\text {int }}=0.742_{-0.025}^{+0.026}$. Figure 6 shows the 431 objects as open circles, the solid points represent galaxies with $D_{n} 4000 \geqslant 1.44$, and the dashed line represents the result of the Bayesian analysis. The slope $b$ is nearly identical to the value, 0.45, obtained by Blanton et al. (2005a) for SDSS galaxies with Sérsic index $n<2$ and $M_{r}<-18$.

In their model for $p\left(\mathrm{SB}_{50, R} \mid M_{R}\right)$, Blanton et al. (2005a) allow for an increase in $\sigma_{\text {int }}$ for less luminous galaxies (for results in the
$B$ band see, e.g., de Jong \& Lacey 2000; Cross \& Driver 2002; Driver et al. 2005). Our data do not support such an increase. For galaxies with $M_{R}<-18$ we find $\sigma_{\text {int }}=0.766_{-0.045}^{+0.050}$, very similar to the result for the sample as a whole. The value of $\sigma_{\text {int }}$ is, however, consistent with the SDSS value at $M_{r} \sim-18$. The overall consistency of the SDSS results with the DLS is remarkable.

\subsection{SHELS $S_{0.1}$ Redshift Survey Completeness}

The completeness of SHELS $_{0.1}$ to the limiting apparent magnitude may, in principle, differ from the survey as a whole. Figure 7 shows our approach to estimating the completeness of the SHELS $_{0.1}$ sample. We use a combination of color and surface brightness as a proxy for redshift to evaluate the completeness for SHELS $_{0.1}$ (Kurtz et al. 2007).

The three panels in the left-hand column of Figure 7 refer to the entire SHELS sample with $R<20.3$; the right-hand panels show the SHELS sample with $20.3 \leqslant R<20.6$. All of the panels show the observed SDSS $g-r$ color as a function of the mean DLS surface brightness within the half-light radius, $\mu_{50, R}$. The gray points in the bottom panels represent the entire photometric SHELS sample including galaxies with and without a redshift; the black points in the upper panels show the total number of these galaxies that lack a redshift. The red points in 
Table 2

Low-redshift Candidate List

\begin{tabular}{|c|c|c|c|c|c|c|}
\hline SHELS ID & SDSS ObjID & R.A.2000 & $\operatorname{Decl}_{2} 2000$ & $R$ & $\begin{array}{c}\mu_{50, R} \\
\left(\mathrm{mag} \mathrm{arcsec}^{-2}\right)\end{array}$ & $g-r^{\mathrm{a}}$ \\
\hline $138.7089504+30.9843187$ & 587738948277698932 & $9: 14: 50.148$ & $30: 59: 03.547$ & 20.5556 & 23.3638717 & 0.521 \\
\hline $138.7255980+30.0542613$ & 587738947203760545 & $9: 14: 54.144$ & 30:03:15.341 & 20.5392 & 23.4122087 & 0.507 \\
\hline $138.7264737+30.3206758$ & 588017978876166652 & $9: 14: 54.354$ & $30: 19: 14.433$ & 19.1392 & 23.6712299 & 0.265 \\
\hline $138.7291346+30.4367746$ & 587738947740697008 & $9: 14: 54.992$ & $30: 26: 12.389$ & 20.3627 & 23.0114264 & 0.612 \\
\hline $138.7487849+29.4746605$ & 587738946666758726 & $9: 14: 59.708$ & $29: 28: 28.778$ & 19.9382 & 24.0572414 & 0.498 \\
\hline $138.7539734+29.7394633$ & 588017978339164239 & $9: 15: 00.954$ & $29: 44: 22.068$ & 20.0661 & 22.5720023 & 0.563 \\
\hline $138.7563217+31.0219574$ & 587738948277699044 & $9: 15: 01.517$ & $31: 01: 19.047$ & 20.3730 & 21.775373 & 0.385 \\
\hline $138.7582448+30.9727119$ & 587738948277698992 & $9: 15: 01.979$ & $30: 58: 21.763$ & 20.1276 & 23.2405294 & 0.295 \\
\hline $138.7623082+29.7296108$ & 588017978339164237 & $9: 15: 02.954$ & $29: 43: 46.599$ & 19.5096 & 22.6763703 & 0.389 \\
\hline $138.7648117+31.0037925$ & 587738948277698637 & $9: 15: 03.555$ & $31: 00: 13.653$ & 20.5552 & 22.4070289 & 0.622 \\
\hline
\end{tabular}

Notes. ${ }^{\text {a }}$ The $g-r$ color is the SDSS fiber color.

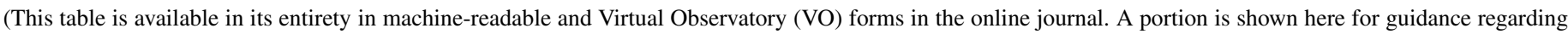
its form and content.)

the central panel indicate galaxies with redshift $z>0.5$ and the blue points indicate galaxies with $z<0.1$, the SHELS $S_{0.1}$ sample. Not surprisingly, galaxies with $20.3 \leqslant R<20.6$ overlap substantially with the $z>0.5$ portion of the redshift survey.

The green line in the central and upper panels is an arbitrary delineation of the boundary of the SHELS ${ }_{0.1}$ sample in magnitude-color space. Ninety-three percent of the apparently brighter SHELS S.1 $_{0.1}$ galaxies with $R<20.3$ with redshifts are blueward of the line and $87 \%$ of the fainter galaxies with $20.3 \leqslant R<20.6$ are blueward of the line. We use this admittedly arbitrary line to estimate the incompleteness of SHELS $_{0.1}$. The black points in the upper panels show all of the SHELS galaxies without redshifts. To estimate the completeness of SHELS $\mathrm{S}_{0.1}$ we estimate the fraction of these black points that are probably galaxies with $z<0.1$. Note that most of the black points, regardless of apparent magnitude, correspond to red galaxies, most probably at high redshift.

Table 2 lists the galaxy candidates below the green line and without redshifts; these are the objects most likely to be missing from our low-redshift sample. For $R<20.3,21 \%$ of the galaxies with redshifts and below the green line are at $z \leqslant 0.1$; for $20.3 \geqslant R<20.6$ this fraction is $11 \%$. Note that the gray points below the green line in the middle and upper panels have redshifts between 0.1 and 0.5 . Only the black points lack a redshift and statistically we expect that most of these are at $z>0.1$. In fact, assuming that the fraction with $z \leqslant 0.1$ is the same among the galaxies without measured redshifts (black points), we expect that only $\sim 14$ of the 86 objects in the table are at $z \leqslant 0.1$. We note that many of the objects in Table 2 are near the edges of the field (particularly the higher surface brightness objects).

To estimate the completeness of SHELS $S_{0.1}$, we compute the fraction of SHELS redshifts with $z<0.1$ both above and below the fiducial green line. We note that these estimates are insensitive to the exact position of the green line. We then assume that these same fractions of the galaxies without redshifts are probably at $z<0.1$. For $R<20.3$, SHELS $S_{0.1}$ is $98 \%$ complete. The differential completeness in the interval $20.3<R<20.6$ for SHELS $_{0.1}$ is $92 \%$, greater than the $89 \%$ for the entire SHELS sample because most of the objects without redshifts are small, faint, and red. Photometric redshifts from SDSS substantiate this analysis. In summary, we estimate that SHELS $_{0.1}$ is $97 \%$ complete to $R=20.6$; we are missing only $14 \pm 4$ objects. Because our samples are substantially complete, we make no corrections for incompleteness in our calculation of the luminosity function.

\section{THE GALAXY LUMINOSITY FUNCTION}

The $\mathrm{SHELS}_{0.1}$ survey probes a small region of the universe to a faint, uniform limiting observed surface brightness. Figure 8 shows the redshift of each survey galaxy as a function of the observed DLS $R$-band surface brightness within the half-light radius, $\mu_{50, R}$. Large-scale structure in the region is obvious in the highly clustered redshift distribution. The distribution of observed surface brightness reaches the survey limit at every redshift.

Figure 8 also provides some insight into the galaxy populations. Again, we segregate the galaxy populations based on the spectroscopic indicator $D_{n} 4000$. Open circles indicate galaxies with a predominantly young population and with $D_{n} 4000<$ 1.44; the solid circles denote galaxies with $D_{n} 4000 \geqslant 1.44$.

In SHELS $_{0.1}$ galaxies dominated by an old population are rare and they tend to be higher surface brightness objects. They appear predominantly in the densest structure in the survey at $z \sim 0.062$, a reflection of the standard morphology-density relation. The general absence of LSB red objects does not result from selection. In fact, the $R$-band DLS photometric data are actually more sensitive to these objects than to LSB blue objects.

The lowest surface brightness galaxies are generally also the lowest luminosity objects (Figure 6). Thus Figure 8 underscores the result previously obtained by Blanton et al. (2005a): the faint end of the luminosity function is dominated by LSB galaxies dominated by a young stellar population. These galaxies are usually blue. It is interesting that even though we select our galaxies at $R$, there are no galaxies with predominantly old populations and $\mu_{50, R} \gtrsim 23.5 \mathrm{mag} \operatorname{arcsec}^{-2}$. As a result of $(1+z)^{4}$ and $K$-dimming, the lowest surface brightness objects in Figure 2 are at $z>0.1$ and thus do not appear in Figure 8.

We compute the luminosity function for SHELS $_{0.1}$ with $0.02 \leqslant z \leqslant 0.1$ (532 galaxies) and for three subsets of this sample separated by rest-frame surface brightness within the half-light radius, $\mathrm{SB}_{50, R}$. The restriction to $z>0.02$ reduces the effect of peculiar velocities on the determination of the luminosity function and sets a low-luminosity limit on the luminosity function of $M_{r}=-13.3+5 \log h$.

The lowest luminosity galaxies in the SHELS $S_{0.1}$ are at $z \lesssim$ 0.015 and do not enter into the luminosity function calculation. Figure 9 shows the five lowest luminosity galaxies listed in 


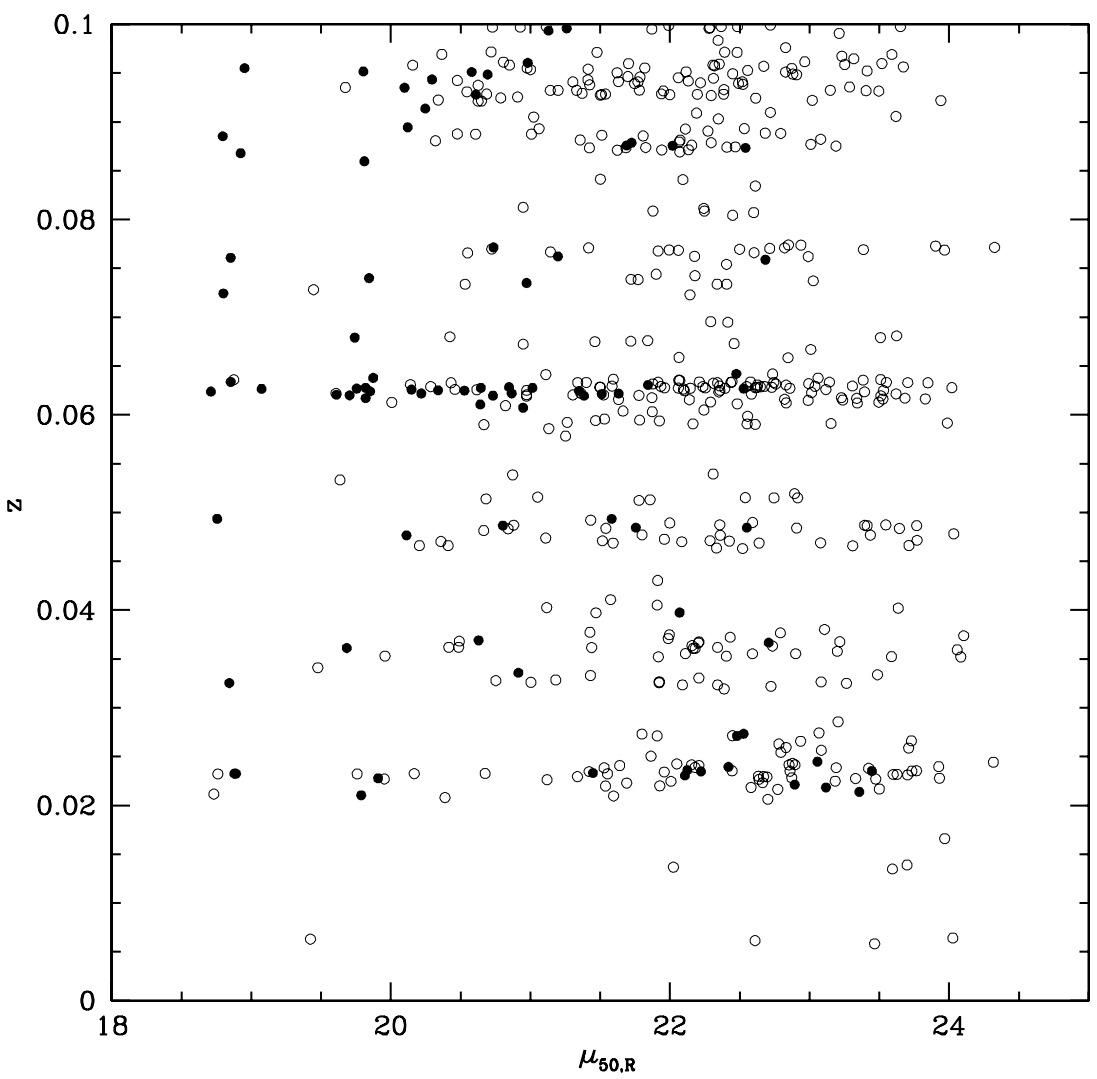

Figure 8. Mean observed surface brightness, $\mu_{50, R}$, as a function of redshift for $\mathrm{SHELS}_{0.1}$ galaxies. Solid points indicate galaxies with $D_{n} 4000>1.44$; open circles indicate objects with $D_{n} 4000 \leqslant 1.44$. This division corresponds well with division by color or spectroscopic type. Note that the large $D_{n} 4000$ (redder) galaxies are predominantly in the densest structure at $z \sim 0.06$.

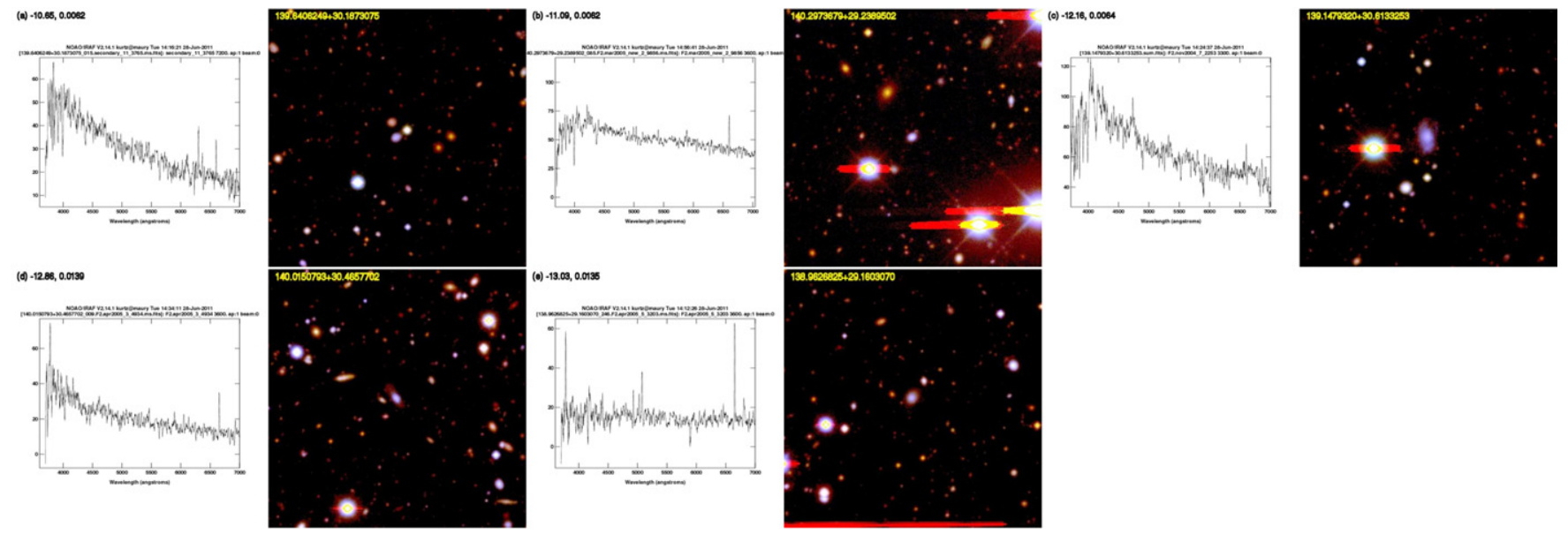

Figure 9. Five lowest luminosity galaxies in SHELS ordered in $R$-band total luminosity. Galaxy (a) has an apparent magnitude $R=20.69$, fainter than the SHELS ${ }_{0.1}$ limit. The bold numbers give the absolute $R$-band luminosity $(h=1)$ and the redshift; the yellow numbers in the 3 arcmin square images give the right ascension and declination of the galaxy. Galaxies (b)-(e) have $R<20.6$. The objects are all blue and their spectra show Balmer absorption and/or $\mathrm{H} \alpha$ emission.

(A color version of this figure is available in the online journal.)

Table 1. Galaxies (b)-(e) all have $R<20.6$ and their rest-frame mean surface brightness within the half-light radius $\mathrm{SB}_{50, R}>$ $22.5 \mathrm{mag} \operatorname{arcsec}^{-2}$. Galaxy (a), the lowest luminosity galaxy in our sample, is just fainter than the SHELS $S_{0.1}$ magnitude limit; it has $R=20.69$. Not surprisingly, these galaxies are blue. Many of the spectra show Balmer absorption characteristic of a predominantly young stellar population. Most of the spectra show $\mathrm{H} \alpha$ emission.

Table 3 gives the number of galaxies in each of the samples we analyze. The high surface brightness (HSB) sample includes all galaxies with $\mathrm{SB}_{50, R}<21.82 \mathrm{mag} \operatorname{arcsec}^{-2}$, the median for the sample. The LSB sample includes the galaxies with $\mathrm{SB}_{50, R} \geqslant 21.82 \mathrm{mag} \operatorname{arcsec}^{-2}$. We note that Blanton et al. (2005a) explore the impact of dividing their sample by surface brightness (Figure 21); they divide their sample at $\mu_{50, r}=21 \sim$ $\mathrm{SB}_{50, R}=20.7 \mathrm{mag} \operatorname{arcsec}^{-2}$, a higher surface brightness by nearly a magnitude $\operatorname{arcsec}^{-2}$ than the median surface brightness for SHELS $_{0.1}$.

To explore the dependence on surface brightness more fully we also compute the luminosity function for galaxies with 

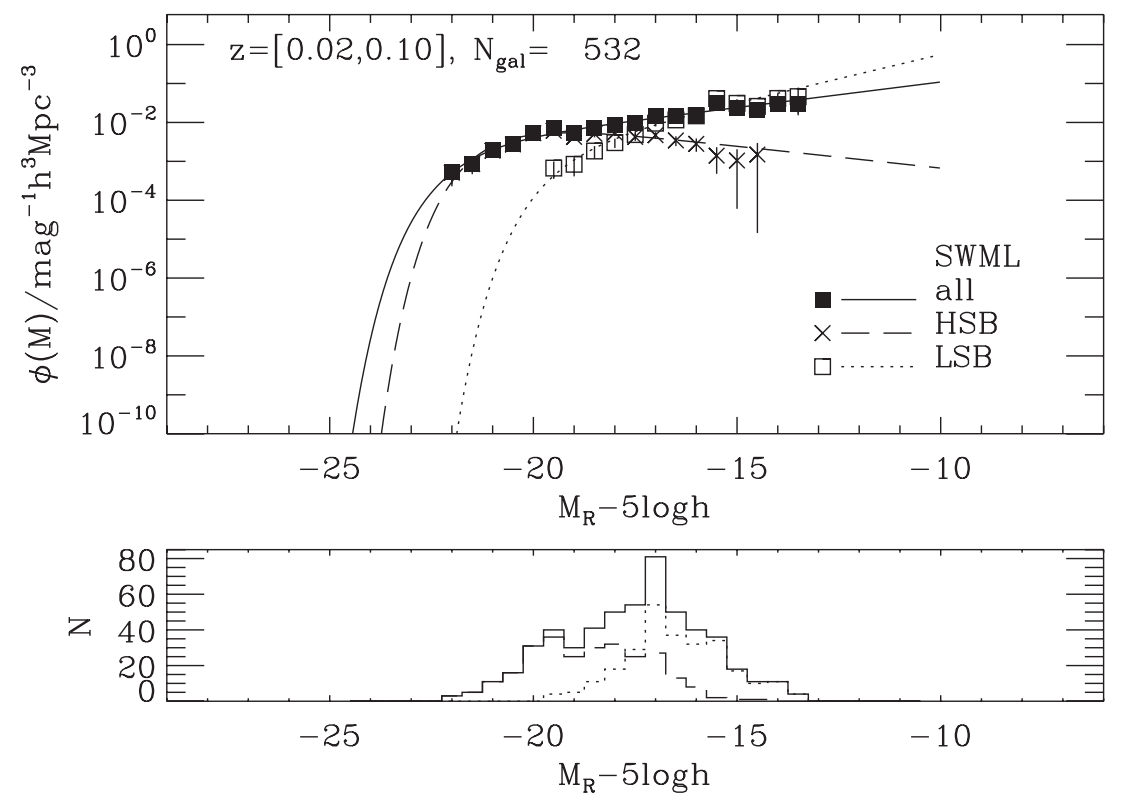

Figure 10. SHELS ${ }_{0.1}$ luminosity functions for all galaxies (solid squares and solid line), for HSB galaxies with $\mathrm{SB}_{50, R}<21.82 \mathrm{mag} \operatorname{arcsec}^{-2}$ (x's and dashed line), and for LSB galaxies with $\mathrm{SB}_{50, R} \geqslant 21.82 \mathrm{mag} \operatorname{arcsec}^{-2}$ (open squares and dotted line). The symbols show the SWML values; the lines are the best-fit Schechter function. The histograms show the number of galaxies in each 0.5 mag bin for each luminosity function.

Table 3

Luminosity Functions

\begin{tabular}{lcccc}
\hline \hline & All & HSB & LSB & SB $_{50, R}>22.5$ \\
\hline$N$ & 532 & 266 & 266 & 135 \\
$\alpha$ & $-1.31 \pm 0.04$ & $-0.69 \pm 0.07$ & $-1.57 \pm 0.09$ & $-1.52 \pm 0.16$ \\
$M_{R}^{*}-5 \log h$ & $-21.32 \pm 0.30$ & $-20.42 \pm 0.15$ & $-18.72 \pm 0.34$ & $-17.32 \pm 0.34$ \\
$\phi^{*} / 10^{-3} \mathrm{mag}^{-1} h^{3} \mathrm{Mpc}^{-3}$ & $4.22 \pm 0.96$ & $11.7 \pm 1.6$ & $4.7 \pm 2.0$ & $8.5 \pm 4.4$ \\
$\chi_{v}^{2}$ & 1.28 & 0.71 & 1.34 & 0.85 \\
$\nu$ & 16 & 14 & 11 & 9 \\
$\mathcal{L} / 10^{8} h L_{\odot} \mathrm{Mpc}^{-3}$ & $0.96 \pm 0.35$ & $0.80 \pm 0.25$ & $0.15 \pm 0.08$ & $0.07 \pm 0.04$ \\
\hline
\end{tabular}

$\mathrm{SB}_{50, R} \geqslant 22.5 \mathrm{mag} \operatorname{arcsec}^{-2}$. This subsample, $\mathrm{SB}_{50, R}>$ $22.5 \mathrm{mag} \operatorname{arcsec}^{-2}$, satisfies the useful definition of LSB galaxies given by O'Neil (2002): the central surface brightness is a magnitude fainter than the night sky. To compute our limit we took the $20 \%$ percentile darkest night sky brightness at Gemini, $R=20.4 \mathrm{mag} \operatorname{arcsec}^{-2}$ (http://www.gemini.edu/ sciops/telescopes-and-sites/observing-condition-constraints/ optical-sky-background) as our fiducial value. We model the objects as pure exponential disks to compute our LSB limit, $\mathrm{SB}_{50, R}>22.5 \mathrm{mag} \operatorname{arcsec}^{-2}$. In this simple exponential disk model, the mean surface brightness within the half-light radius (the quantity we use in this study) is 1.1 mag fainter than the central surface brightness.

We apply the well-known stepwise maximum likelihood (SWML) technique (Efstathiou et al. 1988) to compute the luminosity function. We also applied the less widely used (but also non-parametric) $C^{-}$(Lynden-Bell 1971) and LCCP (Takeuchi et al. 2000) techniques to the data; the results are indistinguishable from the SWML results and for simplicity and clarity, we do not report them here.

Figures 10 and 12 show the results of the luminosity function calculation for the entire sample and for the three subsamples. Figures 11 and 13 show the corresponding confidence contours of the luminosity function parameters. The points in the luminosity function plots show the SWML results. We use the bootstrap method to compute the uncertainty at each point. We

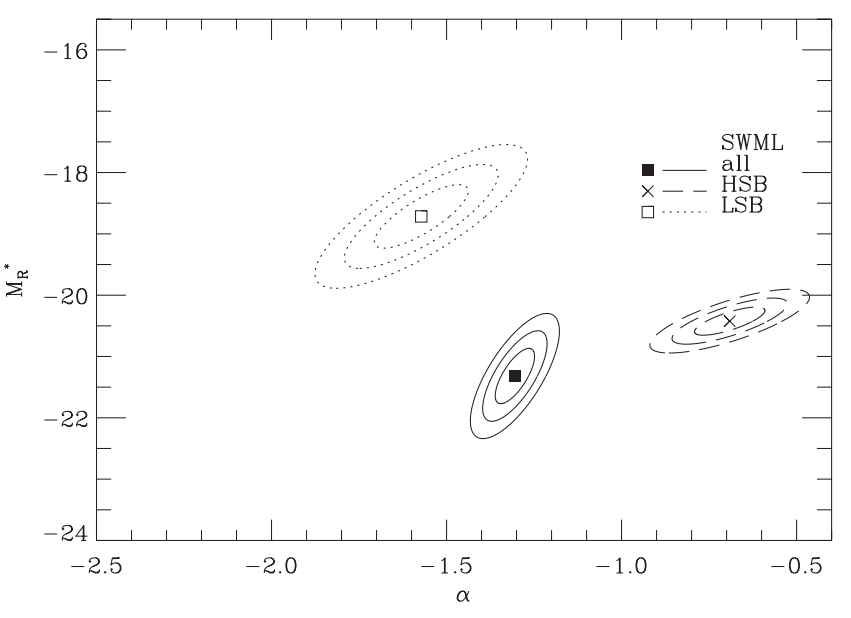

Figure 11. Confidence contours for the SHELS $\mathrm{S}_{0.1}$ luminosity function parameters for all galaxies (solid squares and solid line), for HSB galaxies with $\mathrm{SB}_{50, R}<21.82 \mathrm{mag} \operatorname{arcsec}^{-2}$ ( $\mathrm{x}$ and dashed line), and for LSB galaxies with $\mathrm{SB}_{50, R} \geqslant 21.82 \mathrm{mag} \operatorname{arcsec}^{-2}$ (open square and dotted line). The symbols show the best-fit Schechter parameters. The contours indicate the $1 \sigma, 2 \sigma$, and $3 \sigma$ limit for the parameters.

resample the galaxy sample 50 times for each luminosity function computation.

Of course, the SWML technique does not assume a form for the luminosity function. We represent the SWML results with a 

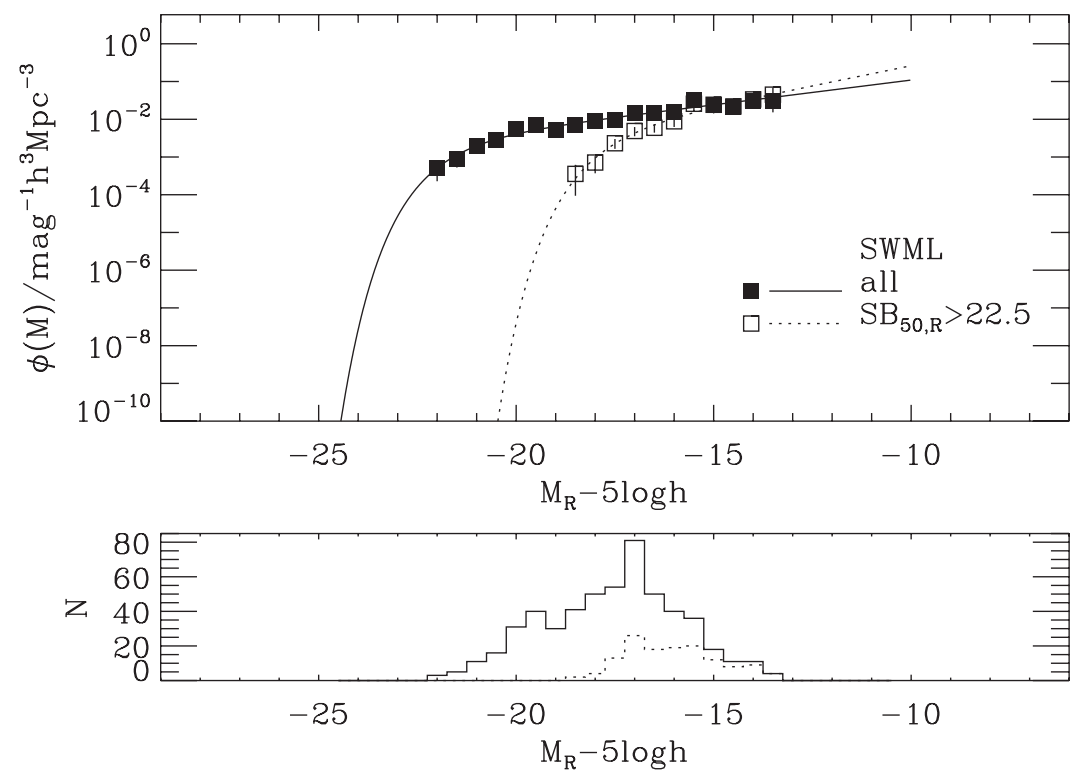

Figure 12. SHELS ${ }_{0.1}$ luminosity functions for the entire SHELS $_{0.1}$ sample (solid squares) compared with the $\mathrm{SB}_{50, R} \geqslant 22.5 \mathrm{mag} \operatorname{arcsec}^{-2}$ subset (open squares), an unusually low surface brightness sample. The histograms show the number of galaxies in each 0.5 mag bin of the respective luminosity functions.

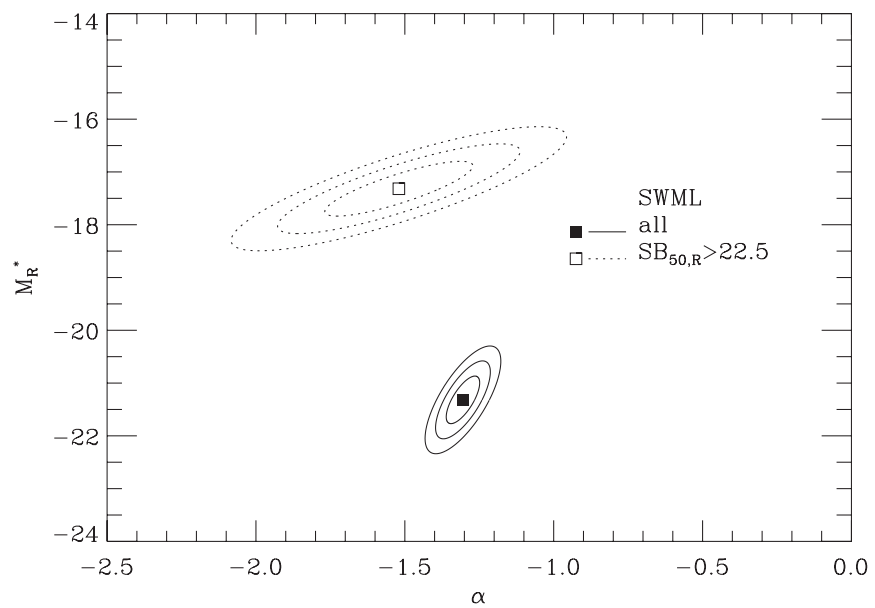

Figure 13. Confidence contours for the sample from Figure 12 with $\mathrm{SB}_{50, R} \geqslant$ $22.5 \mathrm{mag} \operatorname{arcsec}^{-2}$ (dotted contours) and for the entire SHELS $_{0.1}$ sample (solid contours). For the low surface brightness sample the error ellipses admit a wide range of values of $\alpha$ and do not exclude $\alpha=-2$. The best fit is $\alpha=-1.52 \pm 0.15$. These low surface brightness galaxies dominate the determination of the faint-end slope for the luminosity function of the sample as a whole.

fit to a single Schechter (1976) function:

$$
\begin{aligned}
\phi(M) d M= & 0.4 \ln 10 \phi^{*} 10^{0.4(\alpha+1)\left(M^{*}-M\right)} \\
& \times \exp \left[-10^{0.4\left(M^{*}-M\right)}\right] d M,
\end{aligned}
$$

where $\alpha$ is the faint-end slope, $M^{*}$ is the characteristic magnitude, and $\phi^{*}$ is the normalization. With their much larger data set Blanton et al. (2005a) fit a double Schechter function. We obtain reasonable $\chi^{2}$ for the single Schechter function fits (Table 3 ).

Table 3 lists the luminosity function parameters for the entire SHELS $_{0.1}$ sample and for each of the three subsamples. Table 3 also lists the luminosity density for each sample. Application of the parametric STY method (Sandage et al. 1979) to our data yields similar results.

Because the volume of SHELS $_{0.1}$ is small, the bright end of the luminosity function and the values of $M_{R}^{*}$ are poorly constrained and cannot be compared with other estimates; the values of $M_{R}^{*}$ and of the luminosity density, $\mathcal{L}$, are only useful for comparison of different subsamples of the SHELS $_{0.1}$ data. Our intent here is to focus on the faint end of the luminosity function and on the contribution of LSB galaxies to the slope.

For the sample as a whole the faint-end slope, $\alpha=-1.31 \pm$ 0.04 , is reasonably well determined. This result is the same as the $r$-band faint-end slope Blanton et al. (2005a) obtained without correction for missing LSB objects $\left(\alpha_{2}=-1.34 \pm 0.01\right)$. This faint-end slope is also consistent with other measurements of field and cluster luminosity functions derived for lowredshift samples with complete spectroscopy (e.g., Christlein \& Zabludoff 2003; Mahdavi et al. 2005; Rines \& Geller 2008).

The luminosity density for the SHELS 0.1 sample is lower than the value obtained by Blanton et al. (2005a) and others as expected based on the selection of the region. We also note that the $\chi_{v}^{2}$ per degree of freedom is reasonable for the single Schechter function fit; the sample is too small to support the more complex approach of fitting a double Schechter function as in Blanton et al. (2005a).

The luminosity functions for the HSB and LSB subsamples demonstrate that the lower surface brightness galaxies dominate the faint end of the luminosity function. The slope for the HSB subsample is quite shallow, $\alpha_{\mathrm{HSB}}=-0.69 \pm 0.07$. The faint-end slope for the LSB sample is $\alpha_{\mathrm{LSB}}=-1.57 \pm 0.09$. This value is somewhat steeper than the fit Blanton et al. (2005a) obtain for their $r$-band luminosity function corrected for missing LSB galaxies $\left(\alpha_{2}=-1.40 \pm 0.01\right)$.

Because there is little correction for surface brightness incompleteness to our limiting magnitude, the steep faint-end slope is an empirical determination of the impact of LSB galaxies on the luminosity function. We make an additional empirical test of this conjecture by extracting a subsample of SHELS $S_{0.1}$ that lies within $1^{\circ}$ of the field center where the integral completeness of SHELS to $R=20.6$ is $97 \%$ (rather than $96 \%$ in the full area). In this region there are 416 galaxies with $z<0.1$ and we estimate that the completeness of SHELS ${ }_{0.1}$ is $99 \%$ according to the technique demonstrated in Figure 7. In other words we are missing $6 \pm 2$ galaxies. When we recompute the luminosity functions for all of the subsamples in surface brightness considered here, the faint-end slopes agree with those in Table 3 to within $1 \sigma$ for the original sample. 
The value of $\mathrm{M}_{R}^{*}$ for the LSB sample is fainter than for the HSB sample as expected from the correlation between surface brightness and luminosity. It is interesting that the fraction of the luminosity density contributed by the LSB half of the sample is only $16 \%$ to the limiting surface brightness and absolute magnitude we sample.

Our lowest surface brightness sample $\mathrm{SB}_{50, R}>$ $22.5 \mathrm{mag} \operatorname{arcsec}^{-2}$ contains 135 galaxies and thus the error in $\alpha$ is large. However, there is no other published luminosity function derived from a highly complete redshift survey of such LSB galaxies. The faint-end slope is $\alpha_{22.5}=-1.52 \pm 0.16$, consistent with the slope we obtain for the larger LSB sample.

\section{DISCUSSION}

Determination of the faint-end slope of the galaxy luminosity function is sensitive to the inclusion of LSB galaxies. Although these galaxies make a relatively small contribution to the total luminosity density, they dominate the count of objects at low luminosity.

The identification of LSB galaxies from a photometric survey is a challenging problem in itself and the acquisition of a spectroscopic redshift for the lowest surface brightness objects is time-consuming even with a large telescope. One alternative approach is the use of photometric redshifts. Here we compare our results with the luminosity function obtained by Liu et al. (2008) for the redshift range $z=0.02-0.1$ in the $2 \mathrm{deg}^{2}$ COSMOS field. In this redshift bin they compute the faint-end slope to a limiting $M_{V} \sim-12.8$, comparable with our $R$-band limit of $\sim-13.0$ for the same redshift interval.

Liu et al. (2008) use photometric redshifts computed according to the method of Mobasher et al. (2007). To compute the luminosity function, Liu et al. (2008) treat each galaxy as a weighted probability-smoothed luminosity distribution. They apply a modified version of the $1 / V_{\max }$ method and use numerical simulations of their procedure to assess the biases and random errors in their technique.

For their entire sample, Liu et al. (2008) obtain a faintend slope, $\alpha_{\text {COSMOS }}=-1.24 \pm 0.07$, remarkably consistent with our $\alpha=-1.31 \pm 0.04$. Liu et al. (2008) segregate their sample according to spectral energy distributions roughly corresponding to various morphological types. Their ScD+Irr bin is probably the most comparable with the LSB portion of our sample. For this subsample, they obtain $\alpha_{\mathrm{COSMOS}, \mathrm{ScD}+\mathrm{Irr}}=$ $-1.46 \pm 0.07$ in essential agreement with our $\alpha_{\mathrm{LSB}}=-1.57 \pm$ 0.09 . It is impressive that two very different techniques yield such similar results over the same redshift range.

Liu et al. (2008) comment that the faint-end slope in their $z=0.02-0.1$ bin may be "qualitatively" dominated by LSB dwarfs that are not detected in their survey at higher redshift. The SHELS ${ }_{0.1}$ faint-end slope is dominated by LSB dwarfs. Thus the essential agreement of the COSMOS lowredshift faint-end slope with SHELS $_{0.1}$ argues strongly that LSB dwarfs (and not evolution) account for the relatively steeper faint-end slope at the lowest redshifts in the COSMOS sample. The comparison of SHELS S.1 $_{1}$ with the COSMOS results underscores the importance of cleanly defined and measured surface brightness limits in surveys addressing the galaxy luminosity function and its evolution.

\section{CONCLUSION}

Measurement of the faint-end slope of the galaxy luminosity function requires attention to lower surface brightness objects that dominate the count at lower luminosities. This issue is, of course, important at all redshifts and failure to understand surface brightness limits may lead to apparent evolution of the faint end of the luminosity function with redshift. We use the SHELS redshift survey of one of the DLS survey fields to evaluate the faint-end slope at low redshift and to examine its sensitivity to surface brightness. The DLS photometry offers the possibility of identifying galaxies with lower surface brightness (the photometric survey is complete to a mean surface brightness within the half-light radius of $27.0 \mathrm{mag} \operatorname{arcsec}^{-2}$ at $R$ ) and, in carrying out the spectroscopic survey, we paid attention to acquiring redshifts for these lower surface brightness objects. The lowest surface brightness objects we identify by careful inspection of all of the galaxy candidates in the field are above the photometric detection limit. Two-hour integrations with Hectospec on the MMT are adequate to acquire a redshift even for the lowest surface brightness objects in the photometric catalog.

We calculate the faint-end slope in the $R$ band for the subset of SHELS galaxies with redshifts in the range $0.02 \leqslant z<0.1$, SHELS $_{0.1}$. This sample contains 532 galaxies with $R<20.6$ with a median surface brightness within the half-light radius of $\mathrm{SB}_{50, R}=21.82 \mathrm{mag} \operatorname{arcsec}^{-2}$. We estimate that there are only $14 \pm 4$ objects missing from this sample. We used this sample to make one of the few direct measurements of the dependence of the faint end of the galaxy luminosity function on surface brightness.

We compare the properties of the SHELS $_{0.1}$ galaxies with the much larger, but shallower NYU-VAGC derived from the SDSS (Blanton et al. 2005a, 2005b). We show that SHELS S.1 $_{0.1}$ has a fainter median observed surface brightness $\mu_{50, r}$ within the halflight radius. The SHELS $S_{0.1}$ sample thus enables a strong test of the relation between absolute magnitude and surface brightness that Blanton et al. (2005a) use to correct their luminosity function for missing LSB objects. We use a Bayesian approach to evaluate the correlation between magnitude and surface brightness; we derive a slope of $0.46 \pm 0.02$, essentially identical to the slope derived by Blanton et al. (2005a) for galaxies with $M_{r}<-18+5 \log h$. Our $R$-band relation extends to $M_{R} \sim-14+5 \log h$ with essentially constant variance around the mean relation.

We compute the faint-end slope of the luminosity function for the SHELS S.1 $_{0.1}$ sample as a whole and for three subsamples segregated by mean surface brightness within the half-light radius. For the sample as a whole the faint-end slope $\alpha=$ $-1.31 \pm 0.04$, is consistent with both the Blanton et al. (2005a) analysis of the SDSS and the Liu et al. (2008) analysis of the COSMOS field. This consistency is impressive given the very different approaches of these three surveys: SDSS is a large area shallow survey with spectroscopy; COSMOS is a deep photometric survey with an analysis based on photometric redshifts; and SHELS $_{0.1}$ is a dense spectroscopic survey with a $6.5 \mathrm{~m}$ telescope of a photometric catalog derived from $5 \mathrm{hr}$ of integration in better than 0.9 seeing on a $4 \mathrm{~m}$ telescope.

A magnitude-limited sample of 135 galaxies with optical spectroscopic redshifts and with mean surface brightness, $\mathrm{SB}_{50, R} \geqslant 22.5 \mathrm{mag} \operatorname{arcsec}^{-2}$ is unique to SHELS $\mathrm{S}_{0.1}$. The faintend slope is $\alpha_{22.5}=-1.52 \pm 0.16$, consistent with previous limits on similarly LSB populations from independent samples. Because SHELS $S_{0.1}$ samples a low-density region of the universe by construction, these LSB objects are predominantly blue. Surveying a larger volume to the depth of SHELS $_{0.1}$ would be an important basis for exploring the dependence of the 
faint end of the luminosity function on environment and galaxy type.

We thank Scott Kenyon for many insightful discussions. We thank Beth Willman for a discussion about Local Group dwarfs. We also thank Michael Strauss and Marijn Franx for encouraging us to use the SHELS survey as a basis for studying the local luminosity function. We thank Michael Blanton and his collaborators for providing the NYU-VAGC on line and we thank Martyn Plummer for his publicly available JAGS code. Perry Berlind and Michael Calkins operated the Hectospec efficiently and with extraordinary expertise. We thank Susan Tokarz for her dedication to careful data reduction. Nelson Caldwell deftly manages Hectospec queue scheduling for optimal scientific results. We thank the anonymous referee for urging us to improve the clarity of the presentation here. This paper makes use of data from the Sloan Digital Sky Survey. The Smithsonian Institution partially supported this research. NSF grant AST-0708433 supports Ian Dell'Antonio's research at Brown University. INFN grant PD51 and the PRIN-MIUR2008 grant 2008NR3EBK_003 "Matter-antimatter asymmetry, dark matter, and dark energy in the LHC era" partially support Antonaldo Diaferio.

Facility: MMT(Hectospec)

\section{REFERENCES}

Andreon, S., \& Hurn, M. A. 2010, MNRAS, 404, 1922

Balogh, M. L., Morris, S. L., Yee, H. K. C., Carlberg, R. G., \& Ellingson, E. 1999, ApJ, 527, 54

Benson, A. J., Bower, R. G., Frenk, C. S., et al. 2003, ApJ, 599, 38

Benson, A. J., Lacey, C. G., Baugh, C. M., Cole, S., \& Frenk, C. S. 2002, MNRAS, 333, 156

Blanton, M. R., Lupton, R. H., Schlegel, D. J., et al. 2005a, ApJ, 631, 208

Blanton, M. R., Schlegel, D. J., Strauss, M. A., et al. 2005b, AJ, 129, 2562

Christlein, D., \& Zabludoff, A. I. 2003, ApJ, 591, 764

Cross, N., \& Driver, S. P. 2002, MNRAS, 329, 579

de Jong, R. S., \& Lacey, C. 2000, ApJ, 545, 781

Disney, M., \& Phillipps, S. 1983, MNRAS, 205, 1253
Driver, S. P., Liske, J., Cross, N. J. G., De Propris, R., \& Allen, P. D. 2005, MNRAS, 360, 81

Efstathiou, G., Ellis, R. S., \& Peterson, B. A. 1988, MNRAS, 232, 431

Fabricant, D., Fata, R., Roll, J., et al. 2005, PASP, 117, 1411

Fabricant, D. G., Hertz, E. N., Szentgyorgyi, A. H., et al. 1998, Proc. SPIE, 3355,285

Fabricant, D. G., Kurtz, M. J., Geller, M. J., et al. 2008, PASP, 120, 1222

Geller, M. J., Dell'Antonio, I. P., Kurtz, M. J., et al. 2005, ApJ, 635, L125

Geller, M. J., Kurtz, M. J., Dell'Antonio, I. P., Ramella, M., \& Fabricant, D. G. 2010, ApJ, 709, 832

Grebel, E. K., Gallagher, J. S., III, \& Harbeck, D. 2003, AJ, 125, 1926

Impey, C. D., Sprayberry, D., Irwin, M. J., \& Bothun, G. D. 1996, ApJS, 105, 209

Kauffmann, G., Heckman, T. M., White, S. D. M., et al. 2003, MNRAS, 341 , 33

Kurtz, M. J., Geller, M. J., Fabricant, D. G., Wyatt, W. F., \& Dell'Antonio, I. P. 2007, AJ, 134, 1360

Kurtz, M. J., \& Mink, D. J. 1998, PASP, 110, 934

Liu, C. T., Capak, P., Mobasher, B., et al. 2008, ApJ, 672, 198

Lynden-Bell, D. 1971, MNRAS, 155, 95

Madgwick, D. S., Lahav, O., Baldry, I. K., et al. 2002, MNRAS, 333, 133

Mahdavi, A., Trentham, N., \& Tully, R. B. 2005, AJ, 130, 1502

Mateo, M. L. 1998, ARA\&A, 36, 435

McGaugh, S. S. 1996, MNRAS, 280, 337

Mink, D. J., Wyatt, W. F., Caldwell, N., et al. 2007, in ASP Conf. Ser. 376, Astronomical Data Analysis Software and Systems XVI, ed. R. A. Shaw, F. Hill, \& D. J. Bell (San Francisco, CA: ASP), 249

Mobasher, B., Capak, P., Scoville, N. Z., et al. 2007, ApJS, 172, 117

Muller, G. P., Reed, R., Armandroff, T., Boroson, T. A., \& Jacoby, G. H. 1998 Proc. SPIE, 3355, 577

O’Neil, K. 2002, in ASP Conf. Ser. 254, Extragalactic Gas at Low Redshift, ed. J. S. Mulchaey \& J. Stocke (San Francisco, CA: ASP), 202

Rines, K., \& Geller, M. J. 2008, AJ, 135, 1837

Roll, J. B., Fabricant, D. G., \& McLeod, B. A. 1998, Proc. SPIE, 3355, 324

Sandage, A., Tammann, G. A., \& Yahil, A. 1979, ApJ, 232, 352

Schechter, P. 1976, ApJ, 203, 297

Sprayberry, D., Impey, C. D., Irwin, M. J., \& Bothun, G. D. 1997, ApJ, 482, 104

Takeuchi, T. T., Yoshikawa, K., \& Ishii, T. T. 2000, ApJS, 129, 1

Westra, E., Geller, M. J., Kurtz, M. J., Fabricant, D. G., \& Dell'Antonio, I. 2010, PASP, 122, 1258

White, S. D. M., \& Rees, M. J. 1978, MNRAS, 183, 341

Wittman, D., Dell'Antonio, I. P., Hughes, J. P., et al. 2006, ApJ, 643, 128

Woods, D., Geller, M. J., Kurtz, M. J., et al. 2010, AJ, 139, 1857 